\title{
Mathematics of a sex-structured model for syphilis transmission dynamics
}

\author{
A. B. Gumel ${ }^{1 *}$, J. M. -S. Lubuma ${ }^{2 \dagger}$, O. Sharomi ${ }^{3 \ddagger}$ and Y. A. Terefe ${ }^{2 \S}$ \\ ${ }^{1}$ School of Mathematical and Statistical Sciences, Arizona State University, \\ Tempe, AZ 85287, USA \\ 2 Department of Mathematics and Applied Mathematics, University of Pretoria, \\ Pretoria 0002, South Africa \\ 3 Department of Applied Mathematics and Sciences, \\ Khalifa University of Science and Technology, \\ Abu Dhabi, 127788, UAE
}

\begin{abstract}
Syphilis, a major sexually-transmitted disease, continues to pose major public health burden in both under-developed and developed nations of the world. This study presents a new two-group sex-structured model for assessing the community-level impact of treatment and condom use on the transmission dynamics and control of syphilis. Rigorous analysis of the model shows that it undergoes the phenomenon of backward bifurcation. In the absence of this phenomenon (which is shown to arise due to the re-infection of recovered individuals), the disease-free equilibrium of the model is shown to be globally-asymptotically stable (GAS) when the associated reproduction number is less than unity. Furthermore, the model can have multiple endemic equilibria when the reproduction threshold exceeds unity. Numerical simulations of the model, using data relevant to the transmission dynamics of the disease in Nigeria, show that, with the assumed $80 \%$ condom efficacy, the disease will continue to persist (i.e., remain endemic) in the population regardless of the level of compliance in condom usage by males. Furthermore, detailed optimal control analysis (using Pontraygin's Maximum
\end{abstract}

*email: agumel@asu.edu

${ }^{\dagger}$ Corresponding author email: jean.lubuma@up.ac.za

$\ddagger$ email: oluwaseun.sharomi@kustar.ac.ae

§email: yibeltal.terefe@up.ac.za 
Principle) reveals that, for situations where the cost of implementing the controls (treatment and condom-use) considered in this study is low, channelling resources to a treatment-only strategy is more effective than channelling them to a condom-use only strategy. Furthermore, as expected, the combined condom-treatment strategy provides a higher population-level impact than the treatment-only strategy or the condom-use only strategy. When the cost of implementing the controls is high, the three strategies are essentially equally as ineffective.

Keywords: Syphilis; reproduction number; re-infection; stability; bifurcation.

AMS Subject Classification: 34A34, 37N25, 92B05, 92D30

\section{Introduction}

Syphilis, a sexually-transmitted disease (STD), caused by the bacteria Treponema Pallidum continues to pose a major public health challenge in both the developing and developed parts of the world $[1,2,4,8]$. The disease accounts for about 12-13 million infections globally [8] and close to 200,000 fatalities every year [63] (the incidence rate for syphilis varies from country -to-country, ranging from 3.5 to 30 per 100,000 in countries like Denmark, Germany, The Netherlands and the United Kingdom [10]) (Data for developing countries are hardly available, though we could find some for Nigeria in [11]). Furthermore, studies from the World Health Organization (WHO) show that a significant number of pregnant women (about 2 million) acquire syphilis infection every year [8] (and mother-to-fetus transmission occurs in $80 \%$ of cases $[3,5,6,8]$ ).

Syphilis, tagged the "great imitator" by Sir William Olser [46], spreads through direct contact with sores created by the bacteria. The infection progresses through multiple stages when left untreated, namely primary stage of infection (usually lasting between 3 and 6 weeks), secondary stage (usually lasting between 4 and 10 weeks), latent stage (usually lasting between 10 weeks and 2 years) and the late (tertiary) stage of infection (usually lasting over two years) [10, 27]. The point of syphilis contamination is portrayed by an ulcerative chancre flagging the start of the primary stage of the disease [10]. The secondary stage of infection is characterized by multiple symptoms, especially fever, lymphadenopathy, skin rash and genital or perinea condyloma latum [10]. The latent stage of infection is where damage to internal organ is possible, causing serious health complications which can lead to death [10]. In the latent stage, all clinical manifestations subside and the infection is apparent only on serologic testing [10]. The late (tertiary) stage of infection can manifest as gummatous disease, cardiovascular disease or central nervous system involvement [10]. Syphilis infection can be treated by using antibiotics, such as penicillin, at any stage of the infection [10] (in fact, in its early stages, the disease can be cured with a single injection of this drug. Infected individuals who have syphilis longer than a year may require additional doses). 
Infected individuals who recovered from syphilis infection do not acquire permanent immunity against re-infection $[9,24,35]$ (hence, re-infection and relapse of infected individuals who have recovered from syphilis infection after treatment, can occur at any stages of the infection $[32,61,65]$.

Mathematical and statistical models of infectious diseases have, historically, provided useful insight into the transmission dynamics and control of infectious diseases. However, for syphilis, very few such models have been designed and used to help to understand its complex transmission dynamics and to evaluate the contribution of any proposed interventions before they are implemented [18]. A basic mathematical model that assumes infected individuals acquire temporary immunity only after recovery from the latent and tertiary infections was designed and analysed by Garnett et.al. [18]. Grassly et. al. [38] were able to fit an SIR model for syphilis for more than 65 US cities between 1941-2002 using realistic data set. Another mathematical model that includes inoculation and behavioral patterns as control while emphasizing that secondary and later syphilis infections confer immunity was designed and analysed by Milner and Zhao [25]. Oxman et. al. [28] used multicompartment iterative computer simulation with empirically derived input data to simulate and ascertain behavioral and sociological features necessary to produce epidemic transmission. Iboi and Okuonghae [20] designed and analysed a mathematical model for the transmission dynamics of syphilis that incorporates early and late latent stages of syphilis infection, reversions of early latent syphilis to the primary and secondary stages as well as the three potential outcomes that emanate from the late latent stage of infection while Saad-Roy et.al. [29] developed a deterministic model for syphilis transmission in gay men population. This study presents a new, two-group, deterministic, sex-structured mathematical model for gaining insight into the transmission dynamics of syphilis in a population. The model is based on the following main assumptions:

1. For mathematical convenient, the primary and secondary stages of infection are lumped together (and are referred to as "early stage of infection"). Furthermore, the latent and tertiary stages of infection are lumped together (and are referred to as "late stage of infection").

2. Individuals in both ("early" and "late") disease stages, including those who failed treatment, are assumed to be capable of transmitting the disease.

3. Re-infection and disease relapse in recovered individuals occur $[\mathbf{9}, \mathbf{1 0}, \mathbf{2 4}, \mathbf{3 2}, \mathbf{3 5}, \mathbf{6 1}, \mathbf{6 5}]$. The paper is organized as follows. The model is formulated in Section 2, and its qualitative features are analysed in Section 3. Detailed sensitivity and uncertainty analyses of the reproduction number of the model are carried out in Section 4. Similarly, optimal control analysis is carried out in Section 5. This is followed by discussion and concluding remarks in Section 6 . 


\section{Model Formulation}

The total sexually-active population at time $t$, denoted by $N(t)$, is divided into the total male $\left(N_{m}(t)\right)$ and female $\left(N_{f}(t)\right)$ population. The total female population is further sub-divided into five mutually-exclusive compartments of susceptible females $\left(S_{F}(t)\right)$, infected females in the early stage $\left(I_{F E}(t)\right)$, infected females in the late stage $\left(I_{F L}(t)\right)$, treated females who failed treatment $\left(Q_{F}(t)\right)$ and treated females who recovered from the infection $\left(R_{F}(t)\right)$. Similarly, the total male population is sub-divided into susceptible males $\left(S_{M}(t)\right)$, infected males in the early stage $\left(I_{M E}(t)\right)$, infected males in the late stage $\left(I_{M L}(t)\right)$, treated males who failed treatment $\left(Q_{M}(t)\right)$ and treated males who recovered from the infection $\left(R_{M}(t)\right)$. Thus,

$$
\begin{aligned}
N(t) & =N_{F}(t)+N_{M}(t) \\
N_{F}(t) & =S_{F}(t)+I_{F E}(t)+I_{F L}(t)+Q_{F}(t)+R_{F}(t), \\
N_{M}(t) & =S_{M}(t)+I_{M E}(t)+I_{M L}(t)+Q_{M}(t)+R_{M}(t) .
\end{aligned}
$$

The susceptible populations (for both males and females) are increased by the recruitment of new sexually-active individuals (assumed susceptible) into the population at a rate $\Lambda_{M}\left(\Lambda_{F}\right)$ for males(females). Susceptible males acquire syphilis infection, following effective contact with infected females (i.e., those in the $I_{F E}, I_{F L}$ and $Q_{F}$ classes), at a rate $\lambda_{M}$, given by

$$
\lambda_{M}(t)=\left(1-\epsilon_{M} c_{M}\right) \frac{p_{M} \beta_{M}}{N_{F}(t)}\left[I_{F E}(t)+\nu_{F} I_{F L}(t)+\xi_{F} Q_{F}(t)\right]
$$

Similarly, susceptible females acquire syphilis infection following effective contact with infected males (i.e., those in the $I_{M E}, I_{M L}$ and $Q_{M}$ classes) at a rate

$$
\lambda_{F}(t)=\left(1-\epsilon_{F} c_{F}\right) \frac{p_{F} \beta_{F}}{N_{M}(t)}\left[I_{M E}(t)+\nu_{M} I_{M L}(t)+\xi_{M} Q_{M}(t)\right]
$$

where the parameters $\epsilon_{F}$ and $\epsilon_{M}$ (with $0<\epsilon_{F}, \epsilon_{M}<1$ ) are the efficacy of condom use for females and males, respectively, while $c_{F}$ and $c_{M}$ (with $0<c_{F}, c_{M}<1$ ) represent, respectively, the proportion of females and males that use condoms consistently (i.e., they are compliance proportions). Hence, the terms $\epsilon_{F} c_{F}$ and $\epsilon_{M} c_{M}$ represent the overall effectiveness of condom use for susceptible females and males, respectively. The terms $p_{F}$ and $p_{M}$ are the probabilities of transmitting syphilis from male-to-female and female-to-male per contact, respectively. The parameters $\beta_{F}$ and $\beta_{M}$ are the average number of sexual partners for females and males, respectively. Thus, the terms $\kappa_{F}=\beta_{F} p_{F}$ and $\kappa_{M}=\beta_{M} p_{M}$ represent the effective contact rates for male-to-female and female-to-male transmission of syphilis, respectively. Furthermore, the modification parameters $\nu_{F}$ 
and $\nu_{M}$ account for the assumed variability in the relative infectiousness of individuals in the $I_{F L}$ and $I_{M L}$ classes, in comparison to infected individuals in the $I_{F E}$ and $I_{M E}$ classes for females and males, respectively. Similarly, the modification parameters $\xi_{F}$ and $\xi_{M}$ account for the assumed variability in the relative infectiousness of individuals in the $Q_{F}$ and $Q_{M}$ classes, in comparison to infected individuals in the $I_{F E}$ and $I_{M E}$ classes for females and males, respectively.

Individuals in the $I_{F E}$ and $I_{M E}$ classes progress to the corresponding late stage $\left(I_{F L}\right.$ and $\left.I_{M L}\right)$ classes at rates $\sigma_{F}$ and $\sigma_{M}$, respectively. Males and females are treated at rates $\tau_{M}$ and $\tau_{F}$, respectively. A fraction, $f_{F}\left(f_{M}\right)$, of the treated individuals from the early stage of infection will recover, and move to the $R_{F}\left(R_{M}\right)$ class, while the remaining fraction, $\left(1-f_{F}\right)\left(\left(1-f_{M}\right)\right)$, will fail treatment and move to the $Q_{F}\left(Q_{M}\right)$ class. Individuals in the late stage of infection who eventually show symptoms of the disease are treated at a rate $\alpha_{T} \tau_{F}\left(\alpha_{T} \tau_{M}\right)$ for females (males), where $\alpha_{T}$ is the modification parameter for the variability in the treatment rate of individuals in the late stage of infection, in comparison to those in the early stage of infection. A fraction, $g_{F}\left(g_{M}\right)$, of the treated individuals in the late stage of infection, will recover and move to the class $R_{F}\left(R_{M}\right)$, while the remaining fraction, $\left(1-g_{F}\right)\left(\left(1-g_{M}\right)\right)$, will fail treatment and move to the $Q_{F}\left(Q_{M}\right)$ class. Individuals who have failed treatment will eventually be treated successfully at a rate $\eta_{T} \tau_{F}\left(\eta_{T} \tau_{M}\right)$ for females (males), and move to the $R_{F}\left(R_{M}\right)$ class. The parameter $\eta_{T}<1$ accounts for the assumed decrease in the recovery rate of individuals in the $Q_{F}$ and $Q_{M}$ classes, in comparison to successfully-treated individuals. Recovered individuals acquire re-infected at rates $\theta_{F} \lambda_{F}(t)$ and $\theta_{M} \lambda_{M}(t)$ for males and females, respectively (with $\theta_{F}>0$ and $\theta_{M}>0$ representing the re-infection parameters for males and females, respectively). Recovered individuals can relapse, and become infectious again, at a rate $\gamma_{F}\left(\gamma_{M}\right)$.

Furthermore, natural mortality occurs in all epidemiological classes at a rate $\mu$, while individuals in the $I_{F L}$ and $I_{M L}$ classes suffer an additional disease-induced mortality at rates $\delta_{F}$ and $\delta_{M}$, for females and males, respectively. Combining all these definitions and assumptions, it follows that the model for the transmission of syphilis in a sexually-active population is given by the following system of differential equations (a flow diagram of the model is depicted in Figure 1, and the state variables and parameters of the model are tabulated in Table 1): 


$$
\begin{aligned}
\frac{d S_{F}}{d t} & =\Lambda_{F}-\left(\lambda_{F}+\mu\right) S_{F} \\
\frac{d I_{F E}}{d t} & =\lambda_{F}\left(S_{F}+\theta_{F} R_{F}\right)+\gamma_{F} R_{F}-\left(\sigma_{F}+\tau_{F}+\mu\right) I_{F E} \\
\frac{d I_{F L}}{d t} & =\sigma_{F} I_{F E}-\left(\alpha_{T} \tau_{F}+\delta_{F}+\mu\right) I_{F L} \\
\frac{d Q_{F}}{d t} & =\left(1-f_{F}\right) \tau_{F} I_{F E}+\left(1-g_{F}\right) \alpha_{T} \tau_{F} I_{F L}-\left(\eta_{T} \tau_{F}+\mu\right) Q_{F}, \\
\frac{d R_{F}}{d t} & =f_{F} \tau_{F} I_{F E}+g_{F} \alpha_{T} \tau_{F} I_{F L}+\eta_{T} \tau_{F} Q_{F}-\theta_{F} \lambda_{F} R_{F}-\left(\mu+\gamma_{F}\right) R_{F}, \\
\frac{d S_{M}}{d t} & =\Lambda_{M}-\left(\lambda_{M}+\mu\right) S_{M}, \\
\frac{d I_{M E}}{d t} & =\lambda_{M}\left(S_{M}+\theta_{M} R_{M}\right)+\gamma_{M} R_{M}-\left(\sigma_{M}+\tau_{M}+\mu\right) I_{M E} \\
\frac{d I_{M L}}{d t} & =\sigma_{M} I_{M E}-\left(\alpha_{T} \tau_{M}+\delta_{M}+\mu\right) I_{M L} \\
\frac{d Q_{M}}{d t} & =\left(1-f_{M}\right) \tau_{M} I_{M E}+\left(1-g_{M}\right) \alpha_{T} \tau_{M} I_{M L}-\left(\eta_{T} \tau_{M}+\mu\right) Q_{M}, \\
\frac{d R_{M}}{d t} & =f_{M} \tau_{M} I_{M E}+g_{M} \alpha_{T} \tau_{M} I_{M L}+\eta_{T} \tau_{M} Q_{M}-\theta_{M} \lambda_{M} R_{M}-\left(\mu+\gamma_{M}\right) R_{M},
\end{aligned}
$$

where $\lambda_{M}$ and $\lambda_{F}$ are as given in Equations (2.1) and (2.2), respectively. For the model (2.3), the following conservation law of sexual contacts holds:

$$
\beta_{F} N_{F}=\beta_{M} N_{M} \text {, so that, } \beta_{M}=\frac{\beta_{F} N_{F}}{N_{M}} \text {. }
$$

The analysis of the model (2.3) will be carried out with $\beta_{M}$ replaced by the equation in (2.4). The model (2.3) complements other models for syphilis transmission dynamics in the literature (such as those in $[18,20,25])$, by, inter alia:

(i) Splitting the total population in terms of gender (males and females).

(ii) Allowing for the re-infection of individuals who recovered from the infection.

(iii) Allowing for the relapse of individuals who recovered from the infection.

The result below holds for the model (2.3).

Theorem 2.1 All solutions of the model system (2.3) with positive initial data remain positive for all time $t>0$. Furthermore, the model is a dynamical system on the region $\Omega=\Omega_{1} \cup \Omega_{2} \subset \mathbb{R}_{+}^{5} \times \mathbb{R}_{+}^{5}$ 


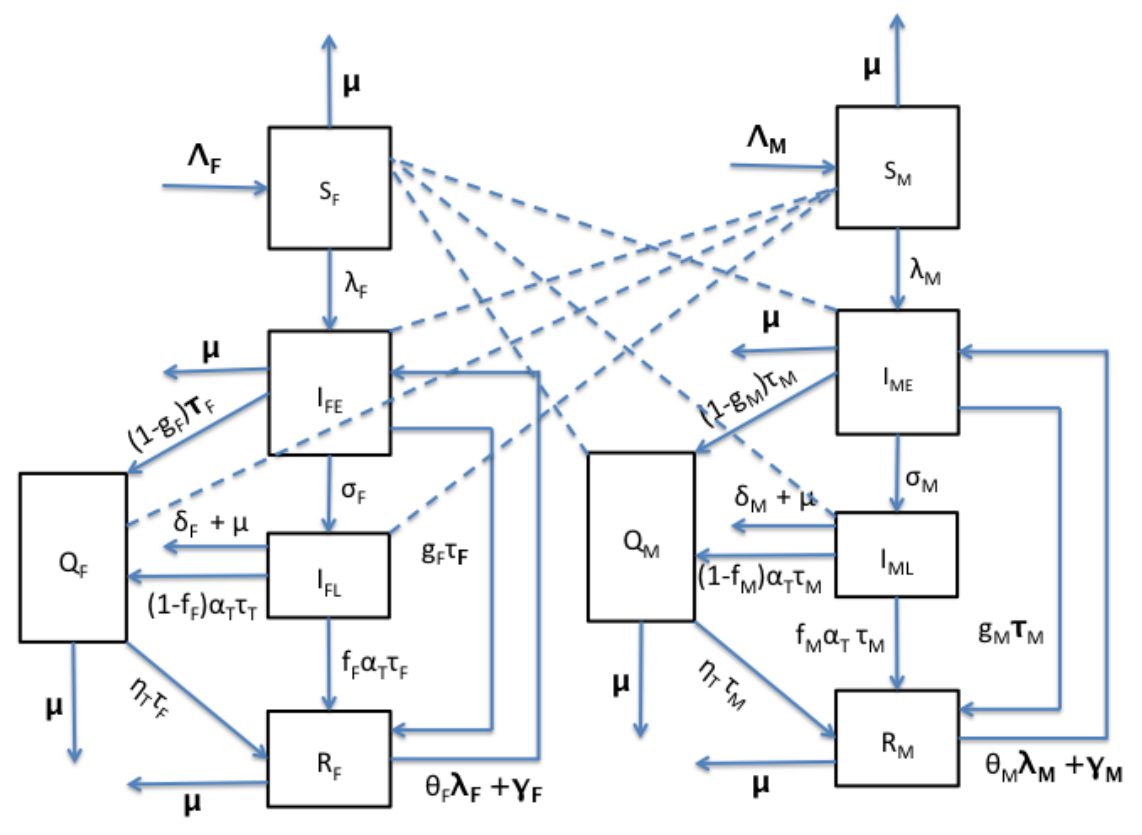

Figure 1: Schematic diagram of the model (2.3)

with,

$$
\begin{gathered}
\Omega_{1}=\left\{\left(S_{F}, I_{F E}, I_{F L}, Q_{F}, R_{F}\right): S_{F}+I_{F E}+I_{F L}+Q_{F}+R_{F}=N_{F} \leq \Lambda_{F} / \mu\right\}, \\
\Omega_{2}=\left\{\left(S_{M}, I_{M E}, I_{M L}, Q_{M}, R_{M}\right): S_{M}+I_{M E}+I_{M L}+Q_{M}+R_{M}=N_{M} \leq \Lambda_{M} / \mu\right\},
\end{gathered}
$$

and the region $\Omega$ is attracting with respect to the model (2.3) with initial conditions in $\mathbb{R}_{+}^{10}$.

The proof of Theorem 2.1 is given in Appendix A. Thus, it is sufficient to consider the dynamics of the flow generated by the model $(2.3)$ in $\Omega([64])$.

\section{Mathematical Analysis}

\subsection{Asymptotic Stability of Disease-free Equilibrium (DFE)}

The DFE of the model (2.3) is given by

$$
\mathcal{E}_{0}=\left(S_{F}^{*}, I_{F E}^{*}, I_{F L}^{*}, Q_{F}^{*}, R_{F}^{*}, S_{M}^{*}, I_{M E}^{*}, I_{M L}^{*}, Q_{M}^{*}, R_{M}^{*}\right)=\left(\frac{\Lambda_{F}}{\mu}, 0,0,0,0, \frac{\Lambda_{M}}{\mu}, 0,0,0,0\right)
$$


The local stability of $\mathcal{E}_{0}$ will be explored using the next generation operator method [34]. Using the notation in $[34,37$, the non-negative matrix, $F$, of new infection terms and the $M$-matrix, $V$, of transition terms associated with the model (2.3) are

$$
F=\left(\begin{array}{cccccccc}
0 & 0 & 0 & 0 & x_{F} & x_{F} \nu_{M} & x_{F} \xi_{M} & 0 \\
0 & 0 & 0 & 0 & 0 & 0 & 0 & 0 \\
0 & 0 & 0 & 0 & 0 & 0 & 0 & 0 \\
0 & 0 & 0 & 0 & 0 & 0 & 0 & 0 \\
x_{M} & x_{M} \nu_{F} & x_{M} \xi_{F} & 0 & 0 & 0 & 0 & 0 \\
0 & 0 & 0 & 0 & 0 & 0 & 0 & 0 \\
0 & 0 & 0 & 0 & 0 & 0 & 0 & 0 \\
0 & 0 & 0 & 0 & 0 & 0 & 0 & 0
\end{array}\right),
$$

and,

$$
V=\left(\begin{array}{cccccccc}
k_{1} & 0 & 0 & -\gamma_{F} & 0 & 0 & 0 & 0 \\
-\sigma_{F} & k_{2} & 0 & 0 & 0 & 0 & 0 & 0 \\
-\left(1-f_{F}\right) \tau_{F} & -\left(1-g_{F}\right) \alpha_{T} \tau_{F} & k_{3} & 0 & 0 & 0 & 0 & 0 \\
-f_{F} \tau_{F} & -g_{F} \alpha_{T} \tau_{F} & -\eta_{T} \tau_{F} & k_{4} & 0 & 0 & 0 & 0 \\
0 & 0 & 0 & 0 & k_{5} & 0 & 0 & -\gamma_{M} \\
0 & 0 & 0 & 0 & -\sigma_{M} & k_{6} & 0 & 0 \\
0 & 0 & 0 & 0 & -\left(1-f_{M}\right) \tau_{M} & -\left(1-g_{M}\right) \alpha_{T} \tau_{M} & k_{7} & 0 \\
0 & 0 & 0 & 0 & -f_{M} \tau_{M} & -g_{M} \alpha_{T} \tau_{M} & -\eta_{T} \tau_{M} & k_{8}
\end{array}\right),
$$

where,

$$
\begin{aligned}
x_{F} & =\frac{\left(1-\epsilon_{F} c_{F}\right) p_{F} \beta_{F} \Lambda_{F}}{\Lambda_{M}}, \quad x_{M}=\frac{\left(1-\epsilon_{M} c_{M}\right) p_{M} \beta_{M} \Lambda_{M}}{\Lambda_{F}} . \\
k_{1} & =\sigma_{F}+\tau_{F}+\mu, \quad k_{2}=\alpha_{T} \tau_{F}+\delta_{F}+\mu, \quad k_{3}=\eta_{T} \tau_{F}+\mu, \quad k_{4}=\mu+\gamma_{F}, \\
k_{5} & =\sigma_{M}+\tau_{M}+\mu, \quad k_{6}=\alpha_{T} \tau_{M}+\delta_{M}+\mu, \quad k_{7}=\eta_{T} \tau_{M}+\mu, \quad k_{8}=\mu+\gamma_{M} .
\end{aligned}
$$

It follows that the basic reproduction number of the model $(2.3)$, denoted by $\mathcal{R}_{0}=\rho\left(F V^{-1}\right.$ ) (where $\rho$ denotes the spectral radius), is given by

$$
\mathcal{R}_{0}=\sqrt{\mathcal{R}_{F} \mathcal{R}_{M}}
$$


where,

$$
\mathcal{R}_{F}=\frac{\left(1-\epsilon_{F} c_{F}\right) p_{F} \beta_{F} k_{4} T_{F}}{T_{F 1}+T_{F 2}} \text { and } \mathcal{R}_{M}=\frac{\left(1-\epsilon_{M} c_{M}\right) p_{M} \beta_{M} k_{8} T_{M}}{T_{M 1}+T_{M 2}}
$$

with,

$$
\begin{aligned}
T_{F} & =\tau_{F} \xi_{F}\left[k_{2}\left(1-f_{F}\right)+\alpha_{T} \sigma_{F}\left(1-g_{F}\right)\right]+k_{3}\left(k_{2}+\nu_{F} \sigma_{F}\right), \\
T_{F 1} & =k_{2}\left\{\mu^{3}+\left[\sigma_{F}+\gamma_{F}+\tau_{F}\left(1+\eta_{T}\right)\right] \mu^{2}+\left[\eta_{T}\left(\tau_{F}+\sigma_{F}+\gamma_{F}\right)+\gamma_{F}\left(1-f_{F}\right)\right] \tau_{F} \mu\right\}, \\
T_{F 2} & =\gamma_{F} \sigma_{F}\left\{\mu^{2}+\left[\delta_{F}+\eta_{T} \tau_{F}+\tau_{F} \alpha_{T}\left(1-g_{F}\right)\right] \mu+\eta_{T} \tau_{F} \delta_{F}\right\}, \\
T_{M} & =\tau_{M} \xi_{M}\left[k_{6}\left(1-f_{M}\right)+\alpha_{T} \sigma_{M}\left(1-g_{M}\right)\right]+k_{7}\left(k_{6}+\nu_{M} \sigma_{M}\right), \\
T_{M 1} & =k_{6}\left\{\mu^{3}+\left[\sigma_{M}+\gamma_{M}+\tau_{M}\left(1+\eta_{T}\right)\right] \mu^{2}+\left[\eta_{T}\left(\tau_{M}+\sigma_{M}+\gamma_{M}\right)+\gamma_{M}\left(1-f_{M}\right)\right] \tau_{M} \mu\right\}, \\
T_{M 2} & =\gamma_{M} \sigma_{M}\left\{\mu^{2}+\left[\delta_{M}+\eta_{T} \tau_{M}+\tau_{M} \alpha_{T}\left(1-g_{M}\right)\right] \mu+\eta_{T} \tau_{M} \delta_{M}\right\} .
\end{aligned}
$$

The result below follows from Theorem 2 in [34].

Lemma 3.1 The DFE of the model (2.3), given by $\mathcal{E}_{0}$, is locally-asymptotically stable (LAS) if $\mathcal{R}_{0}<1$, and unstable if $\mathcal{R}_{0}>1$.

The threshold quantity $\mathcal{R}_{0}$ measures the average number of new syphilis infections generated by a single infected individual in a completely susceptible population $[14,30,34]$. It is the geometric mean of the reproduction numbers for females $\left(\mathcal{R}_{F}\right)$ and males $\left(\mathcal{R}_{M}\right)$. In particular $\mathcal{R}_{F}\left(\mathcal{R}_{M}\right)$ represents the average number of new syphilis infections in the female (male) population generated by a single infected male (female) introduced into a completely susceptible female (male) population. The square root in the expression for $\mathcal{R}_{0}$ in (3.1) is due to the fact that two generations (males-females-males) are needed to complete the syphilis transmission cycle.

Lemma 3.1 implies that syphilis can be effectively controlled in (or eliminated from) the community (when $\mathcal{R}_{0}<1$ ) if the initial sizes of the subpopulations of the model are in the basin of attraction of the $\operatorname{DFE}\left(\mathcal{E}_{0}\right)$. To ensure that disease elimination is independent of the initial sizes of the sub-populations, it is necessary to show that the DFE is globally-asymptotically stable (GAS) if $\mathcal{R}_{0}<1$. This is explored, for a special case, in Section 3.2.2. 


\subsection{Backward Bifurcation Analysis}

\subsubsection{Existence of backward bifurcation}

Before investigating the global asymptotic stability of the DFE, it is instructive to determine the number of equilibrium solutions the model (2.3) can have. Let

$$
\mathcal{E}_{1}=\left(S_{F}^{* *}, I_{F E}^{* *}, I_{F L}^{* *}, Q_{F}^{* *}, R_{F}^{* *}, S_{M}^{* *}, I_{M E}^{* *}, I_{M L}^{* *}, Q_{M}^{* *}, R_{M}^{* *}\right)
$$

be any arbitrary equilibrium of the model (2.3). Furthermore, let

$$
\begin{aligned}
& \lambda_{F}^{* *}=\left(1-\epsilon_{F} c_{F}\right) \frac{p_{F} \beta_{F}}{N_{M}^{* *}}\left(I_{M E}^{* *}+\nu_{M} I_{M L}^{* *}+\xi_{M} Q_{M}^{* *}\right), \\
& \lambda_{M}^{* *}=\left(1-\epsilon_{M} c_{M}\right) \frac{p_{M} \beta_{M}}{N_{F}^{* *}}\left(I_{F E}^{* *}+\nu_{F} I_{F L}^{* *}+\xi_{F} Q_{F}^{* *}\right),
\end{aligned}
$$

be the associated infection rates ("force of infection") for females and males, respectively, at steady-state. To find conditions for the existence of an equilibrium for which syphilis infection is endemic in the population (i.e., the scenario where at least one of $I_{F E}^{* *}, I_{F L}^{* *}, Q_{F}^{* *}, I_{M E}^{* *}, I_{M L}^{* *}$ and $Q_{M}^{* *}$ is non-zero), the equations of the model (2.3) are solved in terms of the aformentioned infection

rates at steady-state $\left(\lambda_{F}^{* *}\right.$ and $\left.\lambda_{M}^{* *}\right)$. Setting the right-hand sides of the equations of the model (2.3) to zero (at steady-state) gives:

$$
\begin{aligned}
S_{F}^{* *} & =\frac{\Lambda_{F}}{\lambda_{F}^{* *}+\mu}, \quad I_{F E}^{* *}=\frac{\lambda_{F}^{* *}\left(a_{11} \lambda_{F}^{* *}+a_{12}\right)}{\left(\lambda_{F}^{* *}+\mu\right)\left(C_{1} \lambda_{F}^{* *}+C_{2}\right)}, \quad I_{F L}^{* *}=\frac{\lambda_{F}^{* *}\left(a_{21} \lambda_{F}^{* *}+a_{22}\right)}{\left(\lambda_{F}^{* *}+\mu\right)\left(C_{1} \lambda_{F}^{* *}+C_{2}\right)}, \\
Q_{F}^{* *} & =\frac{\lambda_{F}^{* *}\left(a_{31} \lambda_{F}^{* *}+a_{32}\right)}{\left(\lambda_{F}^{* *}+\mu\right)\left(C_{1} \lambda_{F}^{* *}+C_{2}\right)}, \quad R_{F}^{* *}=\frac{a_{41} \lambda_{F}^{* *}}{\left(\lambda_{F}^{* *}+\mu\right)\left(C_{1} \lambda_{F}^{* *}+C_{2}\right)}, \\
S_{M}^{* *} & =\frac{\Lambda_{M}}{\lambda_{M}^{* *}+\mu}, \quad I_{M E}^{* *}=\frac{\lambda_{M}^{* *}\left(b_{11} \lambda_{M}^{* *}+b_{12}\right)}{\left(\lambda_{M}^{* *}+\mu\right)\left(D_{1} \lambda_{F}^{* *}+D_{2}\right)}, \quad I_{M L}^{* *}=\frac{\lambda_{M}^{* *}\left(b_{21} \lambda_{M}^{* *}+b_{22}\right)}{\left(\lambda_{M}^{* *}+\mu\right)\left(D_{1} \lambda_{M}^{* *}+D_{2}\right)} \\
Q_{M}^{* *} & =\frac{\lambda_{M}^{* *}\left(b_{31} \lambda_{M}^{* *}+b_{32}\right)}{\left(\lambda_{M}^{* *}+\mu\right)\left(D_{1} \lambda_{M}^{* *}+D_{2}\right)}, \quad R_{M}^{* *}=\frac{b_{41} \lambda_{M}^{* *}}{\left(\lambda_{M}^{* *}+\mu\right)\left(D_{1} \lambda_{M}^{* *}+D_{2}\right)},
\end{aligned}
$$


where,

$$
\begin{aligned}
& a_{11}=k_{2} k_{3} \Lambda_{F} \theta_{F}, \quad a_{12}=k_{2} k_{3} \Lambda_{F} k_{4}, \quad a_{21}=k_{3} \sigma_{F} \Lambda_{F} \theta_{F}, \quad a_{22}=k_{3} \sigma_{F} \Lambda_{F} k_{4}, \\
& a_{31}=\Lambda_{F} \tau_{F}\left[k_{2}\left(1-f_{F}\right)+\sigma_{F} \alpha_{T}\left(1-g_{F}\right)\right] \theta_{F}, \quad a_{32}=\Lambda_{F} \tau_{F}\left[k_{2}\left(1-f_{F}\right)+\sigma_{F} \alpha_{T}\left(1-g_{F}\right)\right] k_{4}, \\
& a_{41}=\Lambda_{F} \tau_{F}\left[\alpha_{T} \eta_{T} \sigma_{F} \tau_{F}\left(1-g_{F}\right)+\eta_{T} \tau_{F} k_{2}\left(1-f_{F}\right)+f_{F} k_{2} k_{3}+g_{F} \alpha_{T} \sigma_{F} k_{3}\right], \quad D_{b}=T_{M 1}+T_{M 2}, \\
& b_{11}=k_{6} k_{7} \Lambda_{M} \theta_{M}, \quad b_{12}=k_{6} k_{7} \Lambda_{M} k_{8}, \quad b_{21}=k_{7} \sigma_{M} \Lambda_{M} \theta_{M}, \quad b_{22}=k_{7} \sigma_{M} \Lambda_{M} k_{8}, \\
& b_{31}=\Lambda_{M} \tau_{M}\left[k_{6}\left(1-f_{M}\right)+\sigma_{M} \alpha_{T}\left(1-g_{M}\right)\right] \theta_{M}, \quad b_{32}=\Lambda_{M} \tau_{M}\left[k_{6}\left(1-f_{M}\right)+\sigma_{M} \alpha_{T}\left(1-g_{M}\right)\right] k_{8}, \\
& b_{41}=\Lambda_{M} \tau_{M}\left[\alpha_{T} \eta_{T} \sigma_{M} \tau_{M}\left(1-g_{M}\right)+\eta_{T} \tau_{M} k_{6}\left(1-f_{M}\right)+f_{M} k_{6} k_{7}+g_{M} \alpha_{T} \sigma_{M} k_{7}\right], \quad C_{b}=T_{F 1}+T_{F 2}, \\
& C_{a}=\theta_{F}\left[k_{1} k_{2} k_{3}+f_{F} k_{2} k_{3} \tau_{F}+g_{F} \alpha_{T} k_{3} \sigma_{F} \tau_{F}-\alpha_{T} \eta_{T} \sigma_{F} \tau_{F}^{2}\left(1-g_{F}\right)-\eta_{T} k_{2} \tau_{F}\left(1-f_{F}\right)\right] \\
& D_{a}=\theta_{M}\left[k_{5} k_{6} k_{7}+f_{M} k_{6} k_{7} \tau_{M}+g_{M} \alpha_{T} k_{7} \sigma_{M} \tau_{M}-\alpha_{T} \eta_{T} \sigma_{M} \tau_{M}^{2}\left(1-g_{M}\right)-\eta_{T} k_{6} \tau_{M}\left(1-f_{M}\right)\right] .
\end{aligned}
$$

Substituting (3.2) with (3.3) into the expressions for $\lambda_{F}^{* *}$ and $\lambda_{M}^{* *}$ in (3.1), and simplifying, gives

$$
\lambda_{M}^{* *}=\frac{\lambda_{F}^{* *}\left(c_{11} \lambda_{F}^{* *}+c_{12}\right)}{c_{13}\left(\lambda_{F}^{* *}\right)^{2}+c_{14} \lambda_{F}^{* *}+c_{15}} \text { and } \lambda_{F}^{* *}=\frac{\lambda_{M}^{* *}\left(c_{21} \lambda_{M}^{* *}+c_{22}\right)}{c_{23}\left(\lambda_{M}^{* *}\right)^{2}+c_{24} \lambda_{M}^{* *}+c_{25}}
$$

where,

$$
\begin{aligned}
& c_{11}=\left(1-\epsilon_{M} c_{M}\right) p_{M} \beta_{M}\left(a_{11}+\nu_{F} a_{21}+\xi_{F} a_{31}\right), \quad c_{12}=\left(1-\epsilon_{M} c_{M}\right) p_{M} \beta_{M}\left(a_{12}+\nu_{F} a_{22}+\xi_{F} a_{32}\right), \\
& c_{13}=a_{11}+a_{21}+a_{31}, \quad c_{14}=\Lambda_{F} C_{a}+a_{12}+a_{22}+a_{32}+a_{41}, \quad c_{15}=\Lambda_{F} C_{b}, \\
& c_{21}=\left(1-\epsilon_{F} c_{F}\right) p_{F} \beta_{F}\left(b_{11}+\nu_{M} b_{21}+\xi_{M} b_{31}\right), \quad c_{22}=\left(1-\epsilon_{F} c_{F}\right) p_{F} \beta_{F}\left(b_{12}+\nu_{M} b_{22}+\xi_{M} b_{32}\right), \\
& c_{23}=b_{11}+b_{21}+b_{31}, \quad c_{24}=\Lambda_{M} D_{a}+b_{12}+b_{22}+b_{32}+b_{41}, \quad c_{25}=\Lambda_{M} D_{b},
\end{aligned}
$$

so that the non-zero (endemic) equilibria of the model (2.3) satisfy:

$$
D_{1}\left(\lambda_{M}^{* *}\right)^{4}+D_{2}\left(\lambda_{M}^{* *}\right)^{3}+D_{3}\left(\lambda_{M}^{* *}\right)^{2}+D_{4} \lambda_{M}^{* *}+D_{5}=0
$$

where,

$$
\begin{aligned}
& D_{1}=c_{13} c_{21}^{2}+c_{14} c_{21} c_{23}+c_{15} c_{23}^{2}, \\
& D_{2}=c_{23}\left(c_{14} c_{22}+2 c_{15} c_{24}\right)+c_{21}\left(c_{14} c_{24}+2 c_{13} c_{22}\right)-c_{11} c_{21}^{2}-c_{12} c_{23} c_{21}, \\
& D_{3}=c_{15}\left(c_{24}^{2}+2 c_{23} c_{25}\right)+c_{22}\left(c_{14} c_{24}-c_{12} c_{23}\right)+c_{13} c_{22}^{2}+c_{21}\left(c_{14} c_{25}-2 c_{11} c_{22}-c_{12} c_{24}\right), \\
& D_{4}=2 c_{24} c_{25} c_{15}+c_{22}\left(c_{14} c_{25}-c_{12} c_{24}\right)-c_{11} c_{22}^{2}-c_{25} c_{12} c_{21}, \\
& D_{5}=c_{15} c_{25}^{2}\left(1-\frac{c_{12} c_{22}}{c_{15} c_{25}}\right)=c_{15} c_{25}^{2}\left(1-\mathcal{R}_{0}^{2}\right) .
\end{aligned}
$$


It can be seen from (3.5) and (3.7) that $D_{1}>0$ (since all the model parameters are non-negative). Furthermore, $D_{5}>0$ whenever $\mathcal{R}_{0}^{2}<1\left(\mathcal{R}_{0}<1\right)$. Thus, the number of possible positive real roots the polynomial (3.6) can have depends on the signs of $D_{2}, D_{3}$ and $D_{4}$. This can be analyzed using the Descartes' Rule of Signs on the quartic $f(x)=D_{1} x^{4}+D_{2} x^{3}+D_{3} x^{2}+D_{4} x+D_{5}$ (with $x=\lambda_{M}^{* *}$ ). Hence, the following results are established.

Theorem 3.2 The model (2.3)

(a) has a unique endemic equilibrium if $\mathcal{R}_{0}>1$ and either of the following holds

(i) $D_{2}>0, D_{3}>0, D_{4}>0$;

(ii) $D_{2}<0, D_{3}<0, D_{4}<0$;

(iii) $D_{2}>0, D_{3}<0, D_{4}<0$;

(iv) $D_{2}>0, D_{3}>0, D_{4}<0$;

(b) could have more than one endemic equilibrium if $\mathcal{R}_{0}>1$ and either of the following holds

(i) $D_{2}<0, D_{3}>0, D_{4}<0$;

(ii) $D_{2}<0, D_{3}<0, D_{4}>0$;

(iii) $D_{2}>0, D_{3}<0, D_{4}>0$;

(iv) $D_{2}<0, D_{3}>0, D_{4}>0$;

(c) could have 2 or more endemic equilibria if $\mathcal{R}_{0}<1$ and any, or all, of $D_{2}, D_{3}$ and $D_{4}$ are negative.

Item (c) of Theorem 3.2 suggests the existence of multiple endemic equilibria when $\mathcal{R}_{0}<1$ (which is typically a signature for the existence of the phenomenon of backward bifurcation [10, 14, 20, 26]). The phenomenon of backward bifurcation, which is characterized by the co-existence of a stable DFE and a stable endemic equilibrium when the associated reproduction number of the model is less than unity, has been observed in numerous disease transmission models, such as those for (or with) vector-borne diseases $[19,30,44,45,47,48,49]$, imperfect vaccination [39, 40, 41, 42, 43], multi-groups $[50,51]$ etc. The presence of this phenomenon in the model $(2.3)$ is now formally explored.

Theorem 3.3 The model (2.3) undergoes a backward bifurcation at $\mathcal{R}_{0}=1$ whenever (where the eigenvectors $v_{2}, v_{7}, w_{2}, w_{3}, w_{4}, w_{5}, w_{7}, w_{8}, w_{9}, w_{10}$ are defined in Appendix B)

$$
\theta_{F}\left(v_{2}-v_{5}\right) w_{5}-v_{2} \sum_{i=2}^{5} w_{i}>0 \text { and } \theta_{M}\left(v_{7}-v_{10}\right) w_{10}-v_{7} \sum_{i=7}^{10} w_{i}>0
$$


The proof of Theorem 3.3, based on using center manifold theory [15, 32], is given in Appendix B (the associated backward bifurcation diagram is depicted in Figure 2). The epidemiological implication of Theorem 3.3 is that the classical epidemiological requirement of having $\mathcal{R}_{0}<1$, while necessary, is no longer sufficient for the effective control of (or elimination of) the disease in the sommonitr

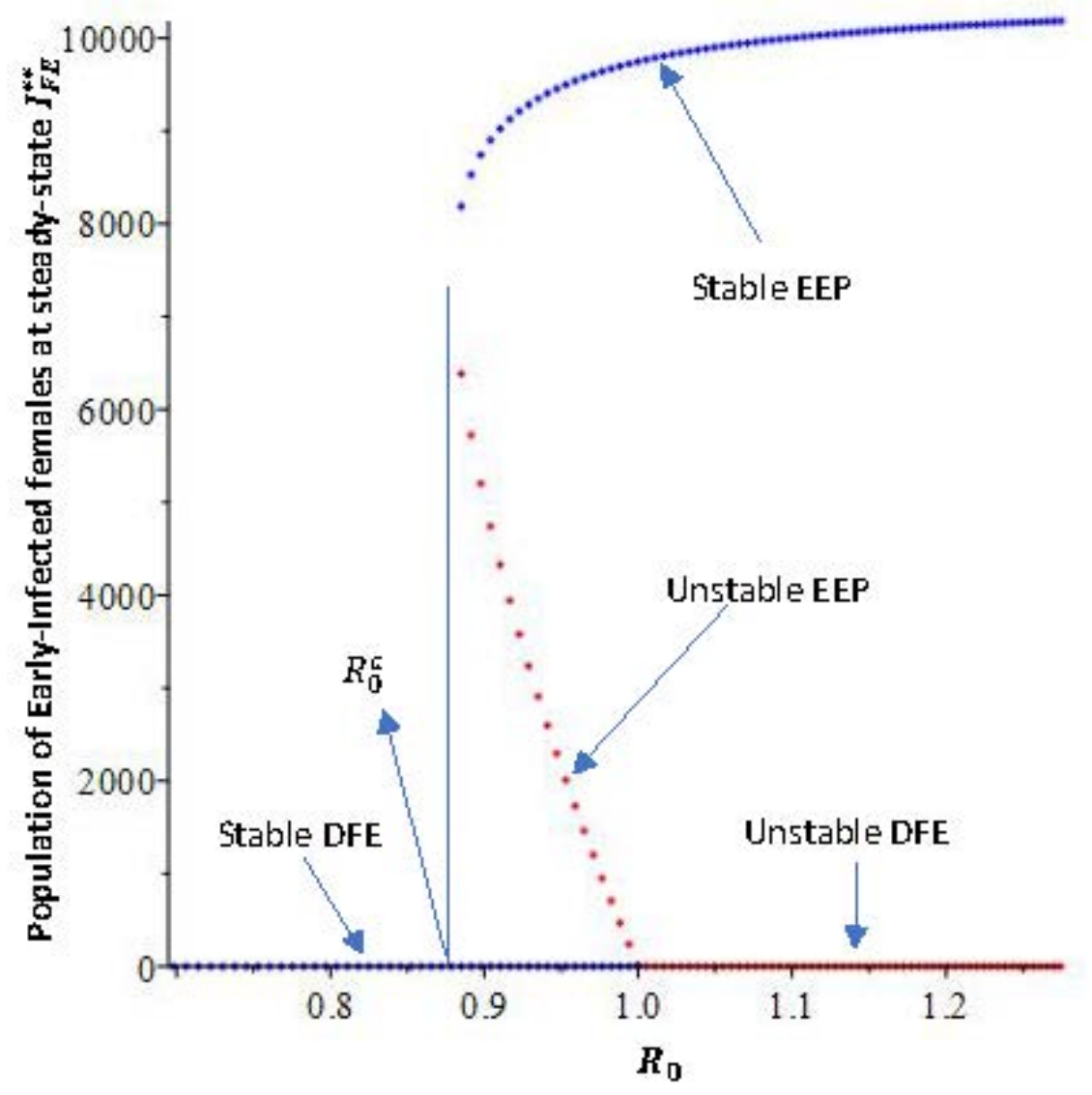

Figure 2: Backward bifurcation diagram of the model (2.3), showing the profile of $I_{F E}^{* *}$ as a function of $\mathcal{R}_{0}$. Parameter values used are: $\Lambda_{F}=160, \mu=0.1 / 70, \beta_{F}=0.085, p_{F}=0.9, c_{F}=0.1, \nu_{F}=$ $1, \xi_{F}=1, \theta_{F}=0.4, \gamma_{F}=0.975, \sigma_{F}=0.012, \tau_{F}=0.975, f_{F}=0.975, g_{F}=0.965, \alpha_{T}=0.08, \eta_{T}=$ $1.5, \epsilon_{F}=0.51, \delta_{F}=10.9, \Lambda_{M}=160, c_{M}=0.1, \nu_{M}=1, \xi_{M}=1, \theta_{M}=0.4, \gamma_{M}=0.975, \sigma_{M}=$ $0.12, \tau_{M}=0.975, \epsilon_{M}=0.91, f_{M}=0.975, g_{M}=0.965, \delta_{M}=10.9, \beta_{M}=\frac{0.085 \Lambda_{F}}{\Lambda_{M}}$ (so that, $p_{M}^{*}=$ 0.2690300627 and $\left.a=5.85501984 \times 10^{5}>0\right)$. 


\subsubsection{Non-existence of backward bifurcation}

Models that incorporate re-infection of recovered individuals are known to lose their backward bifurcation property when the re-infection parameters are set to zero (see, for instance, [19, 30, 51]). In this section, the role of the re-infection of recovered females $\left(\theta_{F}\right)$ and males $\left(\theta_{M}\right)$ on the backward bifurcation phenomenon of the model $(2.3)$ will be investigated. It is convenient to define $\tilde{\mathcal{R}}_{0}=$ $\left.\mathcal{R}_{0}\right|_{\theta_{F}=\theta_{M}=0}$.

Theorem 3.4 The special case of the model (2.3) in the absence of re-infection of recovered individuals (i.e., $\theta_{F}=\theta_{M}=0$ ) does not undergo a backward bifurcation at $\tilde{\mathcal{R}}_{0}=1$.

The proof of Theorem 3.4, based on using center manifold theory, is given in Appendix C. The consequence of Theorem 3.4 is that this study confirms the fact that re-infection (of recovered individuals) does, indeed, cause backward bifurcation in syphilis transmission dynamics. To further

confirm the absence of backward bifurcation in the model (when $\tilde{\mathcal{R}}_{0}<1$ ) for the special case of the model (2.3) with $\theta_{F}=\theta_{M}=0$, a global asymptotic stability result is given for the $\operatorname{DFE}\left(\mathcal{E}_{0}\right)$.

Theorem 3.5 The DFE of the special case of the model (2.3) with $\theta_{F}=\theta_{M}=0$ is GAS in $\Omega$ whenever $\tilde{\mathcal{R}}_{0}<1$.

The proof of Theorem 3.5, based on using a Comparison Theorem [22], is given in Appendix D (the result in Theorem 3.5 is numerically illustrated in Figure 3). The epidemiological implication of Theorem 3.5 is that, in the absence of re-infection (i.e., $\theta_{F}=\theta_{M}=0$ ), syphilis will be effectively controlled in (or eliminated from) the community if the threshold quantity $\tilde{\mathcal{R}}_{0}$ can be brought to (and maintained at) a value less than unity.

\subsection{Existence of Unique Endemic Equilibrium: Special Case}

In this section, the possible existence of a unique endemic equilibrium of the model (2.3) will be explored, for the special case with no re-infection (i.e., $\theta_{F}=\theta_{M}=0$ ). In the absence of re-infection of recovered individuals (i.e., $\left.\theta_{F}=\theta_{M}=0\right)$, the coefficients $D_{i}(i=1 \ldots 5)$ in $(3.7)$ reduce to

$$
\begin{aligned}
& D_{1}=D_{2}=0, \quad D_{3}=c_{15} c_{24}^{2}+c_{22} c_{14} c_{24}, \\
& D_{4}=2 c_{24} c_{25} c_{15}+c_{22}\left(c_{14} c_{25}-c_{12} c_{24}\right), \\
& D_{5}=c_{15} c_{25}^{2}\left(1-\frac{c_{12} c_{22}}{c_{15} c_{25}}\right)=c_{15} c_{25}^{2}\left[1-\left(\tilde{\mathcal{R}}_{0}\right)^{2}\right] .
\end{aligned}
$$




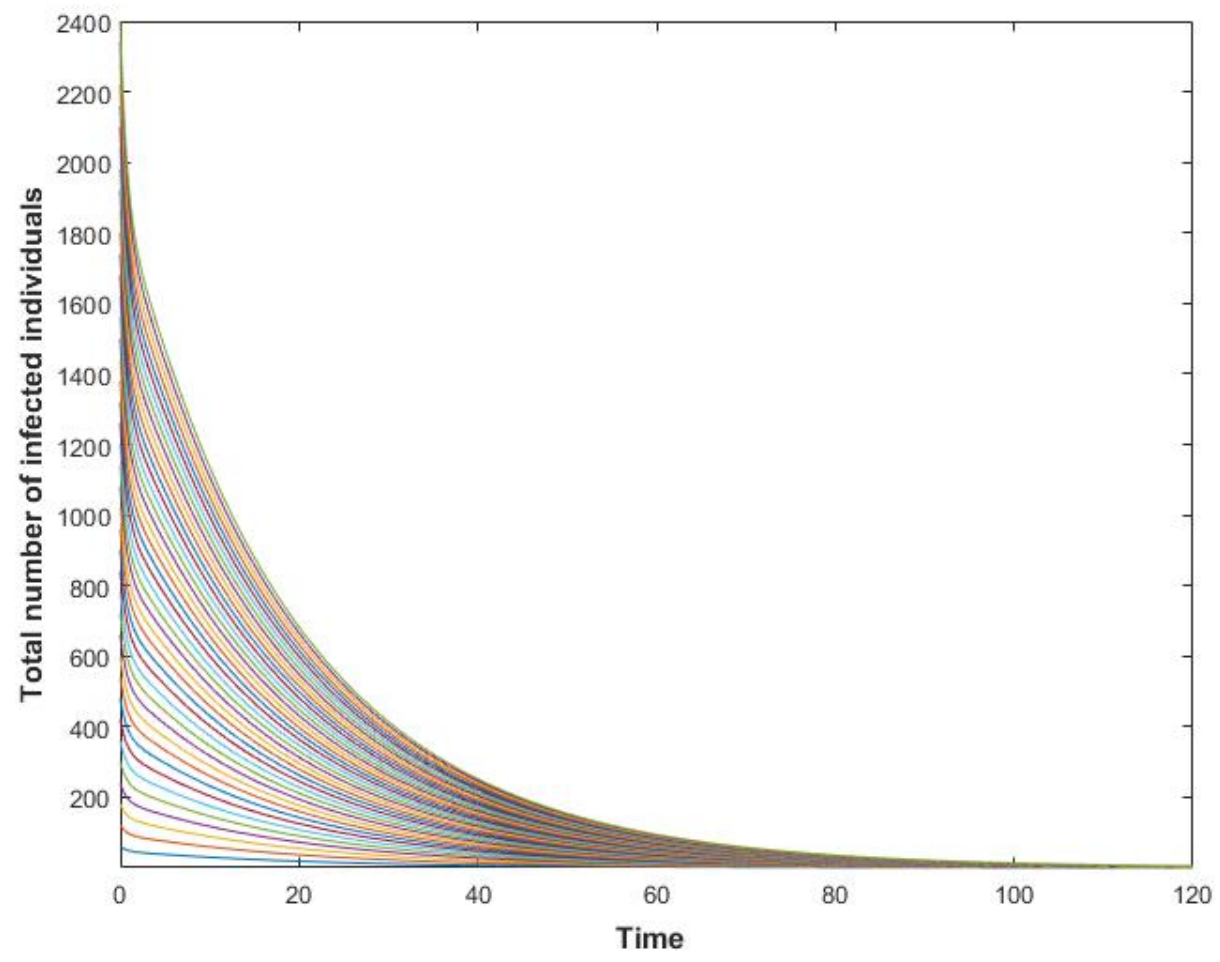

Figure 3: Simulation of the model, showing the total number of infected individuals as a function of time, using multiple initial conditions. Parameter values used are: $\Lambda_{F}=160, \mu=1 / 70, \beta_{F}=$ $0.1, p_{F}=0.9, c_{F}=0.5, \nu_{F}=1, \xi_{F}=1, \theta_{F}=0, \gamma_{F}=0.975, \sigma_{F}=0.12, \tau_{F}=0.5, f_{F}=0.75, g_{F}=$ $0.65, \alpha_{T}=0.08, \eta_{T}=1.5, \epsilon_{F}=0.51, \delta_{F}=0.9, \Lambda_{M}=160, c_{M}=0.5, \nu_{M}=1, \xi_{M}=1, \theta_{M}=0, \gamma_{M}=$ $0.975, \sigma_{M}=0.12, \tau_{M}=0.5, \epsilon_{M}=0.91, f_{M}=0.75, g_{M}=0.65, \delta_{M}=0.9, \beta_{M}=\frac{0.1 \Lambda_{F}}{\Lambda_{M}}$ (so that, $\left.\tilde{\mathcal{R}}_{0}=0.3975<1\right)$.

Thus, the polynomial (3.6) now becomes

$$
D_{3}\left(\lambda_{M}^{* *}\right)^{2}+D_{4} \lambda_{M}^{* *}+D_{5}=0
$$

Solving the quadratic (3.9) gives the roots $\lambda_{M}^{* *}=-\frac{c_{25}}{c_{24}}<0$ and $\lambda_{M}^{* *}=\frac{c_{15} c_{25}\left[\left(\tilde{\mathcal{R}}_{0}\right)^{2}-1\right]}{c_{15} c_{24}+c_{14} c_{22}}<0$ whenever $\left(\tilde{\mathcal{R}}_{0}\right)^{2}<1\left(\tilde{\mathcal{R}}_{0}<1\right)$. Hence, in this case (with $\left.\theta_{F}=\theta_{M}=0\right)$, no endemic equilibrium exists whenever $\left(\tilde{\mathcal{R}}_{0}\right)^{2}<1\left(\tilde{\mathcal{R}}_{0}<1\right)$. Further, for $\left(\tilde{\mathcal{R}}_{0}\right)^{2}>1\left(\tilde{\mathcal{R}}_{0}>1\right)$, the roots $\lambda_{M}^{* *}=-\frac{c_{25}}{c_{24}}<0$ and $\lambda_{M}^{* *}=\frac{c_{15} c_{25}\left[\left(\tilde{\mathcal{R}}_{0}\right)^{2}-1\right]}{c_{15} c_{24}+c_{14} c_{22}}>0$ confirm the existence of a unique endemic equilibrium when $\tilde{\mathcal{R}}_{0}>1$. This result is summarised below.

Theorem 3.6 In the absence of re-infection of recovered individuals (i.e., $\theta_{F}=\theta_{M}=0$ ), the model (2.3) has a unique endemic equilibrium whenever $\tilde{\mathcal{R}}_{0}>1$, and no endemic equilibrium 
otherwise.

The result below follows from Theorem 3.4, Theorem 3.6 and Item (iv) of Theorem 4.1 in $[15]$.

Theorem 3.7 The unique endemic equilibrium of the special case of the model (2.3), with $\theta_{F}=$ $\theta_{M}=0$, is LAS whenever $\hat{\mathcal{R}}_{0}>1$ and $\hat{\mathcal{R}}_{0}$ near 1 .

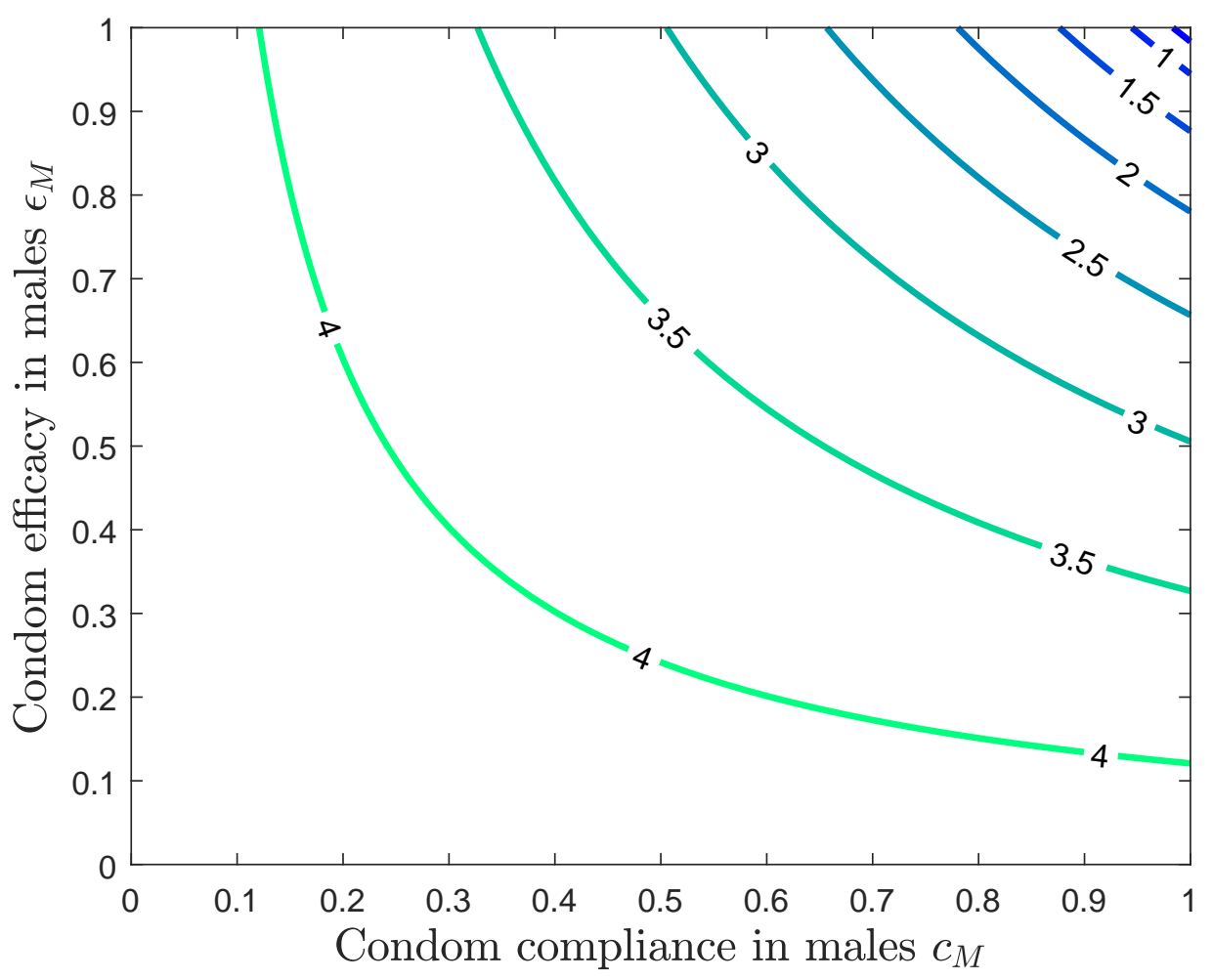

Figure 4: Contour plot of $\hat{\mathcal{R}}_{0}$ as a function of condom compliance $\left(c_{M}\right)$ and efficacy $\left(\epsilon_{M}\right)$ in males.

\section{Uncertainty and Sensitivity Analysis}

The asymptotic analysis of most epidemiological models have dependably been based on the threshold quantity called the basic reproduction number, which can lead to uncertainties particularly when the model consists of many parameters. Another reason for uncertainties in these models is the absence of precision in the estimation of all associated parameters, error in collecting and interpreting data and natural variations. Thus, to determine the impact of the model parameters on 
the transmission dynamics of the syphilis, an uncertainty and sensitivity analysis will be conducted using Latin Hypercube Sampling (LHS) and Partial Rank Correlation Coefficients (PRCCs) [60].

LHS is a statistical method for generating a sample of plausible collections of parameter values from a multidimensional distribution [60]. Using the range of plausible values for the thirty-one parameters of the model (2.3) (see Table 2 for the mean and associated distributions of these parameters), a sample of size $n=10,000$ is obtained through LHS. LHS treats the model inputs (i.e., the model parameters), as random variables. Thus, appropriate probability distributions will be associated with each parameter. The analysis below assumes that the model inputs can take the form of uniform, gamma or normal distributions. The LHS procedure is implemented by dividing the range of values for each given parameter into equally probable, intervals and one selected at random from each interval with the objective of uniformly filling the input space [60].

PRCC is a robust sensitivity measure for nonlinear but monotonic relationships between input and output, as long as little to no correlation exists between the inputs [54]. PRCC is considered to be more powerful at determining the sensitivity of a parameter that is strongly monotonic yet highly nonlinear [55]. The analysis below uses PRCCs to assess the sensitivity of the output $\left(\mathcal{R}_{0}\right)$ to that of the model input parameters. The magnitude, as well as the statistical significance, of the PRCC value of a parameter indicates that parameters contribution to the models prediction imprecision [60]. The parameters with large PRCC values $(>0.5$ or $<-0.5)$ are the most important [56]. Thus, a PRCC approaching -1 to +1 indicates a strong effect of the corresponding parameter to $\mathcal{R}_{0}$. The sign indicates the qualitative relationship between the parameter inputs and $\mathcal{R}_{0}$. A negative sign indicates that the LHS parameter is inversely proportional to the $\mathcal{R}_{0}$.

The analysis is completed using parameter values relevant to syphilis transmission dynamics in Nigeria [11], given in Table 2. The distribution of the mean value of $\mathcal{R}_{0}$ as a function of distributions of various parameters of the model reveals that the estimate of $\mathcal{R}_{0}$ for syphilis is approximately 4.44 with $95 \%$ CI. The probability that $\mathcal{R}_{0}>1$ for syphilis is given by 1 . Hence, under the assumed conditions, syphilis will become endemic in the population.

Results from the sensitivity analyses of the model, using $\mathcal{R}_{0}$ as the response function, show (Figure 5) that the top five dominant parameters of the model are effective contact rate for females $\left(\kappa_{F}=\beta_{F} p_{F}\right)$ and males $\left(\kappa_{M}=\beta_{M} p_{M}\right)$, proportion of treated females(males) in the early stage who recovered from infection $\left(f_{F}\left(f_{M}\right)\right)$, condom compliance in males $\left(c_{M}\right)$ and the modification parameter for the assumed decrease in the recovery rate of individuals in the $Q_{F}$ and $Q_{M}$ classes, in comparison to successfully-treated individuals $\left(\eta_{T}\right)$. The implication of these analyses is that effective community-wide control of the disease can be achieved by minimizing the contact rates $\kappa_{F}$ and $\kappa_{M}$ (perhaps by implementing an effective public health education campaign to encourage safer sex practices within the sexually-active population), early 
detection and treatment of infected individuals (this can be achieved by devoting resources to monitoring infected individuals via contact tracing), encouraging and increasing condom compliance in the sexually-active male population (this can also be achieved via effective public health education campaign) and increase the recovery rate of individuals who failed treatment (by, perhaps, using better drugs and encouraging treated individuals to adhere to the prescribed treatment regimen). Thus, this study identifies key parameters that should be targeted for the design of effective public health strategy or policy for combatting the spread of syphilis in the community.

\section{Optimal Control Analysis}

In this section, an analysis to determine an optimal intervention strategy, based on the two controls (treatment and compliance to condom use), will be carried out. It is assumed that the treatment rate $\tau_{F}\left(\tau_{M}\right)$ and condom compliance rate $c_{F}\left(c_{M}\right)$ for females (males), are now time-dependent, and will therefore act as the control variables. Using these controls, the model (2.3) now becomes

$$
\begin{aligned}
\frac{d S_{F}}{d t} & =\Lambda_{F}-\left(\lambda_{F}+\mu\right) S_{F}, \\
\frac{d I_{F E}}{d t} & =\lambda_{F}\left(S_{F}+\theta_{F} R_{F}\right)+\gamma_{F} R_{F}-k_{1} I_{F E}, \\
\frac{d I_{F L}}{d t} & =\sigma_{F} I_{F E}-k_{2} I_{F L}, \\
\frac{d Q_{F}}{d t} & =\left(1-f_{F}\right) \tau_{F}(t) I_{F E}+\left(1-g_{F}\right) \alpha_{T} \tau_{F}(t) I_{F L}-k_{3} Q_{F}, \\
\frac{d R_{F}}{d t} & =f_{F} \tau_{F}(t) I_{F E}+g_{F} \alpha_{T} \tau_{F}(t) I_{F L}+\eta_{T} \tau_{F}(t) Q_{F}-\theta_{F} \lambda_{F} R_{F}-k_{4} R_{F}, \\
\frac{d S_{M}}{d t} & =\Lambda_{M}-\left(\lambda_{M}+\mu\right) S_{M}, \\
\frac{d I_{M E}}{d t} & =\lambda_{M}\left(S_{M}+\theta_{M} R_{M}\right)+\gamma_{M} R_{M}-k_{5} I_{M E}, \\
\frac{d I_{M L}}{d t} & =\sigma_{M} I_{M E}-k_{6} I_{M L}, \\
\frac{d Q_{M}}{d t} & =\left(1-f_{M}\right) \tau_{M}(t) I_{M E}+\left(1-g_{M}\right) \alpha_{T} \tau_{M}(t) I_{M L}-k_{7} Q_{M}, \\
\frac{d R_{M}}{d t} & =f_{M} \tau_{M}(t) I_{M E}+g_{M} \alpha_{T} \tau_{M}(t) I_{M L}+\eta_{T} \tau_{M}(t) Q_{M}-\theta_{M} \lambda_{M} R_{M}-k_{8} R_{M},
\end{aligned}
$$




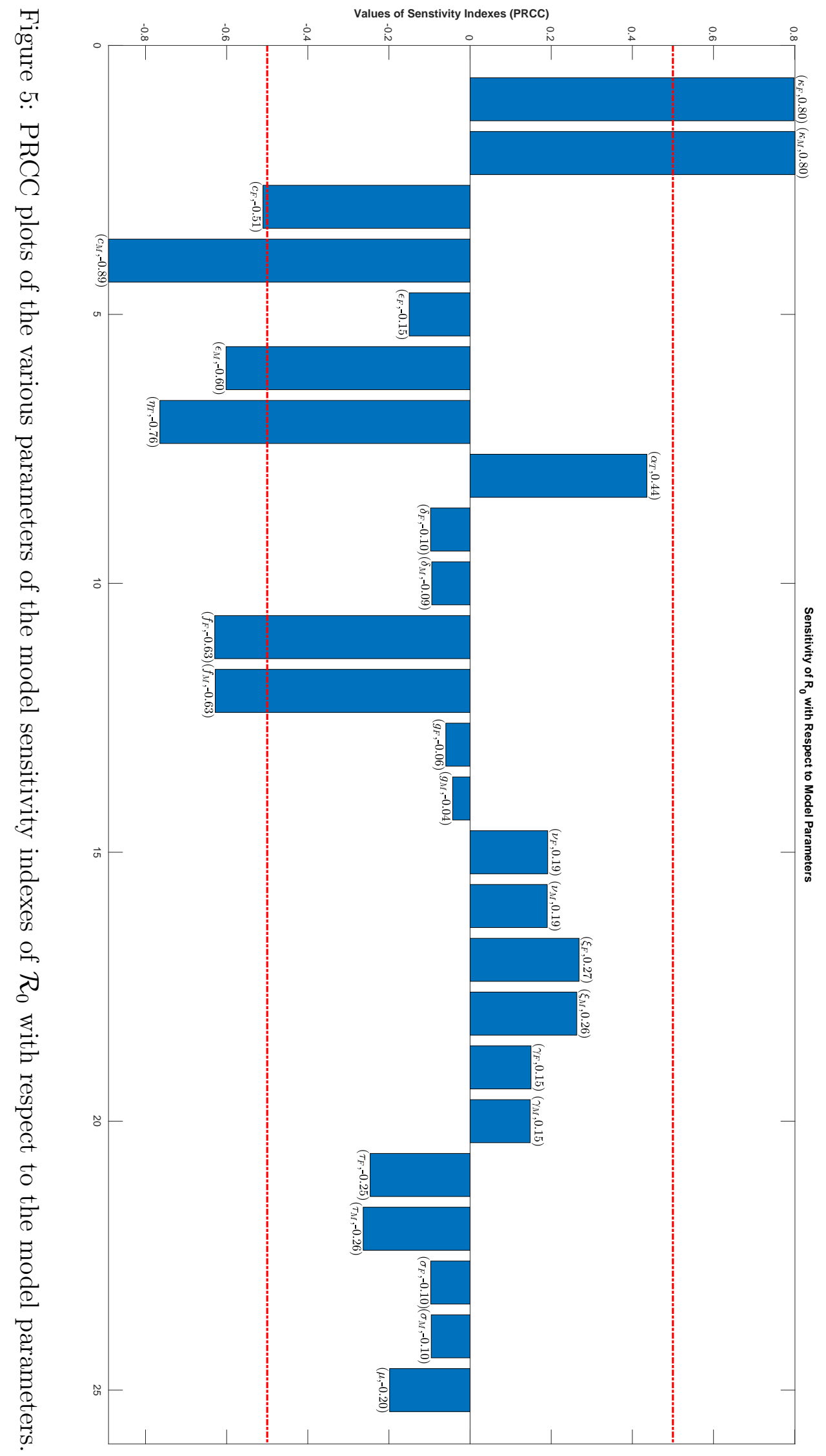


where,

$$
\begin{aligned}
\lambda_{F}(t) & =\left[1-\epsilon_{F} c_{F}(t)\right] \frac{p_{F} \beta_{F}}{N_{M}(t)}\left[I_{M E}(t)+\nu_{M} I_{M L}(t)+\xi_{M} Q_{M}(t)\right], \quad \beta_{M}=\frac{\beta_{F} N_{F}}{N_{M}}, \\
\lambda_{M}(t) & =\left[1-\epsilon_{M} c_{M}(t)\right] \frac{p_{M} \beta_{M}}{N_{F}(t)}\left[I_{F E}(t)+\nu_{F} I_{F L}(t)+\xi_{F} Q_{F}(t)\right], \\
k_{1} & =\sigma_{F}+\tau_{F}(t)+\mu, \quad k_{2}=\alpha_{T} \tau_{F}(t)+\delta_{F}+\mu, \quad k_{3}=\eta_{T} \tau_{F}(t)+\mu, \quad k_{4}=\mu+\gamma_{F}, \\
k_{5} & =\sigma_{M}+\tau_{M}(t)+\mu, \quad k_{6}=\alpha_{T} \tau_{M}(t)+\delta_{M}+\mu, \quad k_{7}=\eta_{T} \tau_{M}(t)+\mu, \quad k_{8}=\mu+\gamma_{M} .
\end{aligned}
$$

Consider the cost function:

$$
\begin{aligned}
J\left[\tau_{F}, \tau_{M}, c_{F}, c_{M}\right] & =\int_{0}^{T}\left[\omega_{1} I_{F E}+\omega_{2} I_{F L}+\omega_{3} Q_{F}+\omega_{4} I_{M E}+\omega_{5} I_{M L}+\omega_{6} Q_{M}\right. \\
& \left.+\frac{1}{2} \omega_{7}\left[\tau_{F}(t)\right]^{2}+\frac{1}{2} \omega_{8}\left[\tau_{M}(t)\right]^{2}+\frac{1}{2} \omega_{9}\left[c_{F}(t)\right]^{2}+\frac{1}{2} \omega_{10}\left[c_{M}(t)\right]^{2}\right] d t
\end{aligned}
$$

Here, the parameters $\omega_{i},(i=1,2, \ldots, 10)$ are the weight factors to help balance each term in the integrand in (5.2), so that none of the terms dominates. The terms in the integrand in (5.2) are explained as follows:

(i) The term $\omega_{1} I_{F E}+\omega_{2} I_{F L}+\omega_{3} Q_{F}+\omega_{4} I_{M E}+\omega_{5} I_{M L}+\omega_{6} Q_{M}$ represents the cost associated with monitoring infected individuals in all stages.

(ii) The term $\omega_{7}\left[\tau_{F}(t)\right]^{2}+\omega_{8}\left[\tau_{M}(t)\right]^{2}$ represents the cost associated with all forms of treatment for all infected individuals.

(iii) The term $\omega_{9}\left[c_{F}(t)\right]^{2}+\omega_{10}\left[c_{M}(t)\right]^{2}$ represents the cost associated with the public health education campaign to educate the public on safer sex practice (condom compliance; correct and consistent usage).

The aim is to minimize the total number of infected females and males, while ensuring that the cost associated with the controls (condom use and treatment) are minimized. It is assumed that the costs of the controls are nonlinear and take quadratic form. The goal is to find an optimal quadruple $\left(\tau_{F}^{*}(t), \tau_{M}^{*}(t), c_{F}^{*}(t), c_{M}^{*}(t)\right)$, such that

$$
J\left[\tau_{F}^{*}, \tau_{M}^{*}, c_{F}^{*}, c_{M}^{*}\right]=\min _{\tau_{F}, \tau_{M}, c_{F}, c_{M} \in W} J\left[\tau_{F}, \tau_{M}, c_{F}, c_{M}\right]
$$

where the control set $(W)$ is defined as:

$$
\begin{aligned}
W= & \left\{\left(\tau_{F}(t), \tau_{M}(t), c_{F}(t), c_{M}(t)\right) \in L^{1}\left(0, t_{f}\right) \mid a_{11} \leq \tau_{F}(t) \leq a_{12}, a_{21} \leq \tau_{M}(t) \leq a_{22}\right. \\
& \left.b_{11} \leq c_{F}(t) \leq b_{12}, b_{21} \leq c_{M}(t) \leq b_{22}\right\}
\end{aligned}
$$




\subsection{Analysis of Optimal Control}

The Pontryagins Maximum Principle [53] gives the necessary conditions that the optimal control quadruple must satisfy. This principle converts Equations (5.1) and (5.2) into a problem of minimizing pointwise a Hamiltonian $(H)$, with respect to the controls $\tau_{F}, \tau_{M}, c_{F}$ and $c_{M}$, defined by:

$$
\begin{aligned}
H= & \omega_{1} I_{F E}+\omega_{2} I_{F L}+\omega_{3} Q_{F}+\omega_{4} I_{M E}+\omega_{5} I_{M L}+\omega_{6} Q_{M} \\
& +\frac{1}{2} \omega_{7}\left[\tau_{F}(t)\right]^{2}+\frac{1}{2} \omega_{8}\left[\tau_{M}(t)\right]^{2}+\frac{1}{2} \omega_{9}\left[c_{F}(t)\right]^{2}+\frac{1}{2} \omega_{10}\left[c_{M}(t)\right]^{2}+\lambda_{S_{F}} g_{1}+\lambda_{I_{F E}} g_{2} \\
& +\lambda_{I_{F L}} g_{3}+\lambda_{Q_{F}} g_{4}+\lambda_{R_{F}} g_{5}+\lambda_{S_{M}} g_{6}+\lambda_{I_{M E}} g_{7}+\lambda_{I_{M L}} g_{8}+\lambda_{Q_{M}} g_{9}+\lambda_{R_{M}} g_{10},
\end{aligned}
$$

where,

$$
\begin{aligned}
g_{1} & =\Lambda_{F}-\left(\lambda_{F}+\mu\right) S_{F}, \\
g_{2} & =\lambda_{F}\left(S_{F}+\theta_{F} R_{F}\right)+\gamma_{F} R_{F}-k_{1} I_{F E}, \\
g_{3} & =\sigma_{F} I_{F E}-k_{2} I_{F L}, \\
g_{4} & =\left(1-f_{F}\right) \tau_{F}(t) I_{F E}+\left(1-g_{F}\right) \alpha_{T} \tau_{F}(t) I_{F L}-k_{3} Q_{F}, \\
g_{5} & =f_{F} \tau_{F}(t) I_{F E}+g_{F} \alpha_{T} \tau_{F}(t) I_{F L}+\eta_{T} \tau_{F}(t) Q_{F}-\theta_{F} \lambda_{F} R_{F}-k_{4} R_{F}, \\
g_{6} & =\Lambda_{M}-\left(\lambda_{M}+\mu\right) S_{M}, \\
g_{7} & =\lambda_{M}\left(S_{M}+\theta_{M} R_{M}\right)+\gamma_{M} R_{M}-k_{5} I_{M E}, \\
g_{8} & =\sigma_{M} I_{M E}-k_{6} I_{M L}, \\
g_{9} & =\left(1-f_{M}\right) \tau_{M}(t) I_{M E}+\left(1-g_{M}\right) \alpha_{T} \tau_{M}(t) I_{M L}-k_{7} Q_{M}, \\
g_{10} & =f_{M} \tau_{M}(t) I_{M E}+g_{M} \alpha_{T} \tau_{M}(t) I_{M L}+\eta_{T} \tau_{M}(t) Q_{M}-\theta_{M} \lambda_{M} R_{M}-k_{8} R_{M},
\end{aligned}
$$

and $\lambda_{S_{F}}, \lambda_{I_{F E}}, \lambda_{I_{F L}}, \lambda_{Q_{F}}, \lambda_{R_{F}}, \lambda_{S_{M}}, \lambda_{I_{M E}}, \lambda_{I_{M L}}, \lambda_{Q_{M}}, \lambda_{R_{M}}$ are adjoint functions. By applying Pontryagin's Maximum Principle [53], and the existence result for the optimal control quadruple $\left(\tau_{F}^{*}(t)\right.$, $\left.\tau_{M}^{*}(t), c_{F}^{*}(t), c_{M}^{*}(t)\right)[52]$, the following result holds:

Theorem 5.1 There exists an optimal control quadruple $\left(\tau_{F}^{*}, \tau_{M}^{*}, c_{F}^{*}, c_{M}^{*}\right)$ and corresponding solution, $S_{F}^{*}, I_{F E}^{*}, I_{F L}^{*}, Q_{F}^{*}, R_{F}^{*}, S_{M}^{*}, I_{M E}^{*}, I_{M L}^{*}, Q_{M}^{*}$ and $R_{F}^{*}$, that minimizes $J\left[\tau_{F}, \tau_{M}, c_{F}, c_{M}\right]$ over $W$. Furthermore, there exists adjoint functions $\lambda_{S_{F}}, \lambda_{I_{F E}}, \lambda_{I_{F L}}, \lambda_{Q_{F}}, \lambda_{R_{F}}, \lambda_{S_{M}}, \lambda_{I_{M E}}, \lambda_{I_{M L}}, \lambda_{Q_{M}}, \lambda_{R_{M}}$, such that

$$
-\frac{d \lambda_{j}}{d t}=\frac{\partial H}{\partial j}
$$


with transversality conditions

$$
\lambda_{j}\left(t_{f}\right)=0 \text {, where } j=S_{F}, I_{F E}, I_{F L}, Q_{F}, R_{F}, S_{M}, I_{M E}, I_{M L}, Q_{M}, R_{M}
$$

Furthermore, the optimal control quadruple $\left(\tau_{F}^{*}, \tau_{M}^{*}, c_{F}^{*}, c_{M}^{*}\right)$ satisfy the optimality conditions

$$
\begin{aligned}
& \tau_{F}^{*}=\min \left\{a_{12}, \max \left(a_{11}, Z_{1}\right)\right\}, \quad \tau_{M}^{*}=\min \left\{a_{22}, \max \left(a_{21}, Z_{2}\right)\right\}, \\
& c_{F}^{*}=\min \left\{b_{11}, \max \left(b_{12}, Z_{3}\right)\right\}, \quad c_{M}^{*}=\min \left\{b_{21}, \max \left(b_{22}, Z_{4}\right)\right\},
\end{aligned}
$$

where,

$$
\begin{aligned}
Z_{1} & =\frac{I_{F E}\left[\lambda_{I_{F E}}-\lambda_{Q_{F}}+f_{F}\left(\lambda_{Q_{F}}-\lambda_{R_{F}}\right)\right]+\alpha_{T} I_{F L}\left(\lambda_{I_{F L}}-\lambda_{Q_{F}}\right)+\left(\alpha_{T} I_{F L} g_{F}+\eta_{T} Q_{F}\right)\left(\lambda_{Q_{F}}-\lambda_{R_{F}}\right)}{\omega_{7}}, \\
Z_{2} & =\frac{I_{M E}\left[\lambda_{I_{M E}}-\lambda_{Q_{M}}+f_{M}\left(\lambda_{Q_{M}}-\lambda_{R_{M}}\right)\right]+\alpha_{T} I_{M L}\left(\lambda_{I_{M L}}-\lambda_{Q_{M}}\right)+\left(\alpha_{T} I_{M L} g_{M}+\eta_{T} Q_{M}\right)\left(\lambda_{Q_{M}}-\lambda_{R_{M}}\right)}{\omega_{8}}, \\
Z_{3} & =\frac{\epsilon_{F} p_{F} \beta_{F}\left(I_{M E}+\nu_{M} I_{M L}+\xi_{M} Q_{M}\right)\left[S_{F}\left(\lambda_{I_{F E}}-\lambda_{S_{F}}\right)+\theta_{F} R_{F}\left(\lambda_{I_{F E}}-\lambda_{R_{F}}\right)\right]}{\omega_{9} N_{M}}, \\
Z_{4} & =\frac{\epsilon_{M} p_{M} \beta_{M}\left(I_{F E}+\nu_{F} I_{F L}+\xi_{F} Q_{F}\right)\left[S_{M}\left(\lambda_{I_{M E}}-\lambda_{S_{M}}\right)+\theta_{M} R_{M}\left(\lambda_{I_{M E}}-\lambda_{R_{M}}\right)\right]}{\omega_{10} N_{F}} .
\end{aligned}
$$

The proof of this theorem is postponed to Appendix E.

\subsection{Numerical Results}

In this section, numerical simulations for the optimal control solution will be carried out using the parameter values in Table 2, and initial data relevant to syphilis transmission dynamics in Nigeria [11]. In particular, we set $S_{F}(0)=91,186,775, I_{F E}(0)=4,582, I_{F L}(0)=366,580, Q_{F}(0)=$ $87,063, R_{F}(0)=0, S_{M}(0)=91,186,775, I_{M E}(0)=4,582, I_{M L}(0)=366,580, Q_{M}(0)=87,063$ and $R_{M}(0)=0$ [11]. The optimal control solution is obtained by solving the optimality system, consisting of 20 ordinary differential equations [62] (i.e., a combination of the equations of the model (2.3) and the associated adjoint equations given in (5.4)). The equations of the model (2.3) are solved forward in time with MATLAB ODE45, first of all, using any initial guess for control. Using the transversality conditions (5.5), the adjoint equations are solved backward in time using the same MATLAB ODE45 routine. This process is done iteratively until the control variables converges. Numerical simulations are then carried out based on the following assumptions:

1. It is assumed that $0 \leq \tau_{F}(t), \tau_{M}(t) \leq 1,0.1 \leq c_{F}(t) \leq 0.3,0.4 \leq c_{M}(t) \leq 0.8$.

2. The weights $\omega_{i}(i=1,2, \ldots, 6)$ are assumed to be equal. This ensures the same cost for monitoring infected and recovered individuals in any class. 
3. The weights $\omega_{7}$ and $\omega_{8}$ are greater than the weights $\omega_{9}$ and $\omega_{10}$. This assumes that the cost associated with treatment $\tau_{F}$ and $\tau_{M}$ exceeds the cost associated with condom compliance $c_{F}$ and $c_{M}$ since treatment usually takes up to 90 days to clear infection.

4. The weights associated with the cost of controls always exceeds that for monitoring individuals in any class (i.e., $\left.\omega_{i}<\omega_{j}, i=1,2, \ldots, 6, j=7,8,9,10\right)$.

The solution profiles of the four optimal controls $\left(\tau_{F}^{*}, \tau_{M}^{*}, c_{F}^{*}\right.$ and $\left.c_{M}^{*}\right)$ are depicted in Figure 6. Furthermore, Figure 7 shows, as expected, a marked reduction in the disease burden in the presence of the optimal control (as against the case with no such control). This figure also shows a corresponding increase in the number of recovered individuals. Further numerical simulations are carried out to illustrate the impact of the cost of implementing the four controls on the number of new cases of infections in females (i.e., $\lambda_{F} S_{F}$ ) and males (i.e., $\lambda_{M} S_{M}$ )generated over a 30-day period. For these simulations, three control strategies are considered, namely the condom-only strategy (where the treatment controls are set to zero), the treatment-only strategy (where the condom controls are set to zero) and the combined treatment-condom strategy. The weights $w_{7}$ and $w_{8}$ correspond to the cost associated with all forms of treatment for all infected females and males whiles, the weights $w_{9}$ and $w_{10}$, corresponding to the cost associated with the public health education campaign to educate the public on safer sex practice (condom compliance; correct and consistent usage), are chosen for this purpose. The weights values 10, 100 and 1000 are considered low as compared to the weights $\left(\omega_{i}(i=1, \ldots, 6)\right)$ which are set to 1 while the weight values $10^{4}, 10^{5}, 10^{6}$ and $10^{7}$ are regarded as high. The results obtained, tabulated in Table 3 , show that, for low cost of controls, the treatment-only strategy resulted in 629,330 new cases, while the condomonly strategy accounted for over 1 million new cases, over the 30-day period. Thus, for situations where control resources are limited, investment in treatment programs is far more effective than investment in condom use as prevention. Furthermore, as expected, the combined treatmentcondom strategy provides an even more effective population-level impact than the treatment-only strategy. For situations where the cost of implementing the controls is high, the three strategies are equally ineffective (close to 2 million new cases). 
(a)

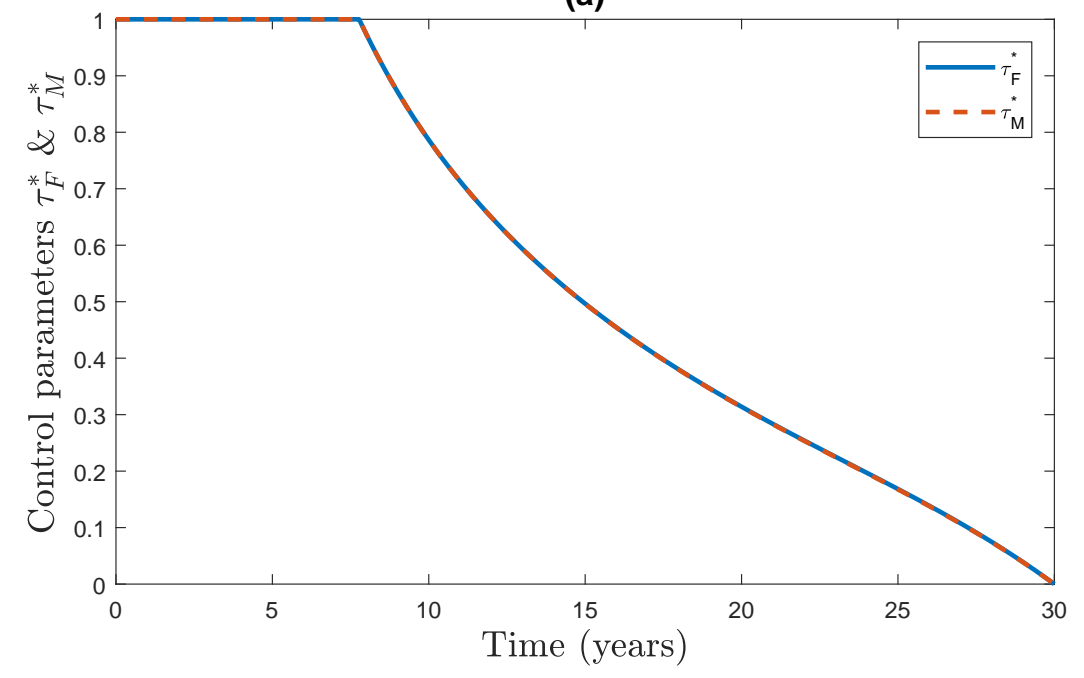

(b)

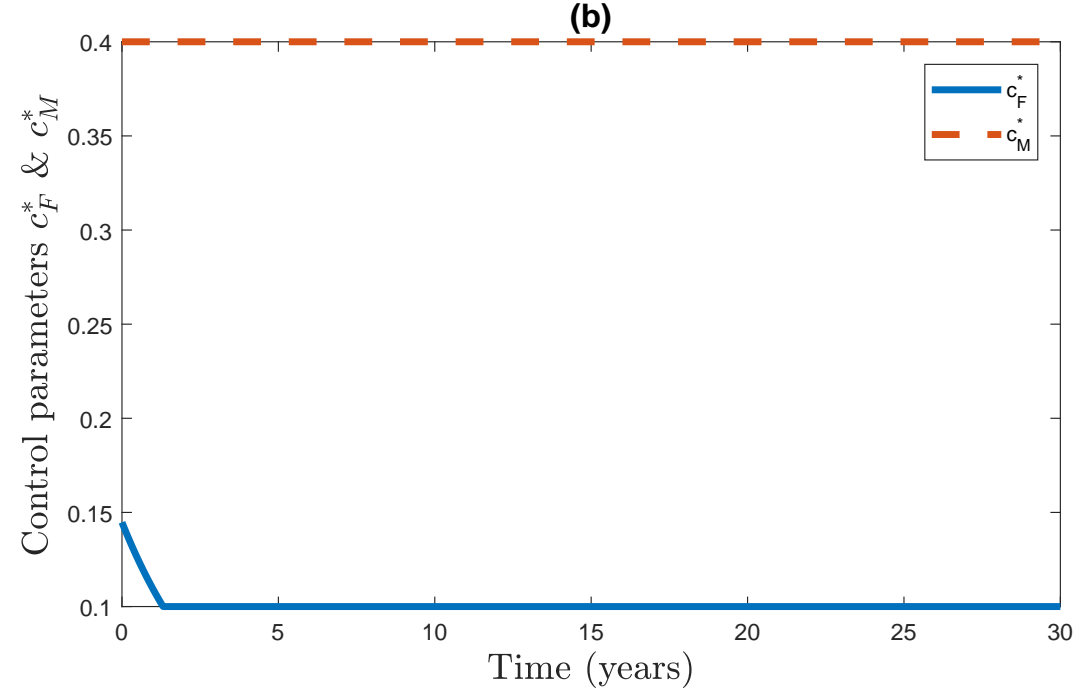

Figure 6: The controls parameters, (a) $\tau_{F}(t)$ and $\tau_{M}(t),(\mathrm{b}) c_{F}(t)$ and $c_{M}(t)$, are plotted as functions of time for $\omega_{i}=1(i=1,2, \ldots, 6), \omega_{7}=\omega_{8}=10^{5}, \omega_{9}=\omega_{10}=10^{4}$. All parameter value used are as given in Table 2. 

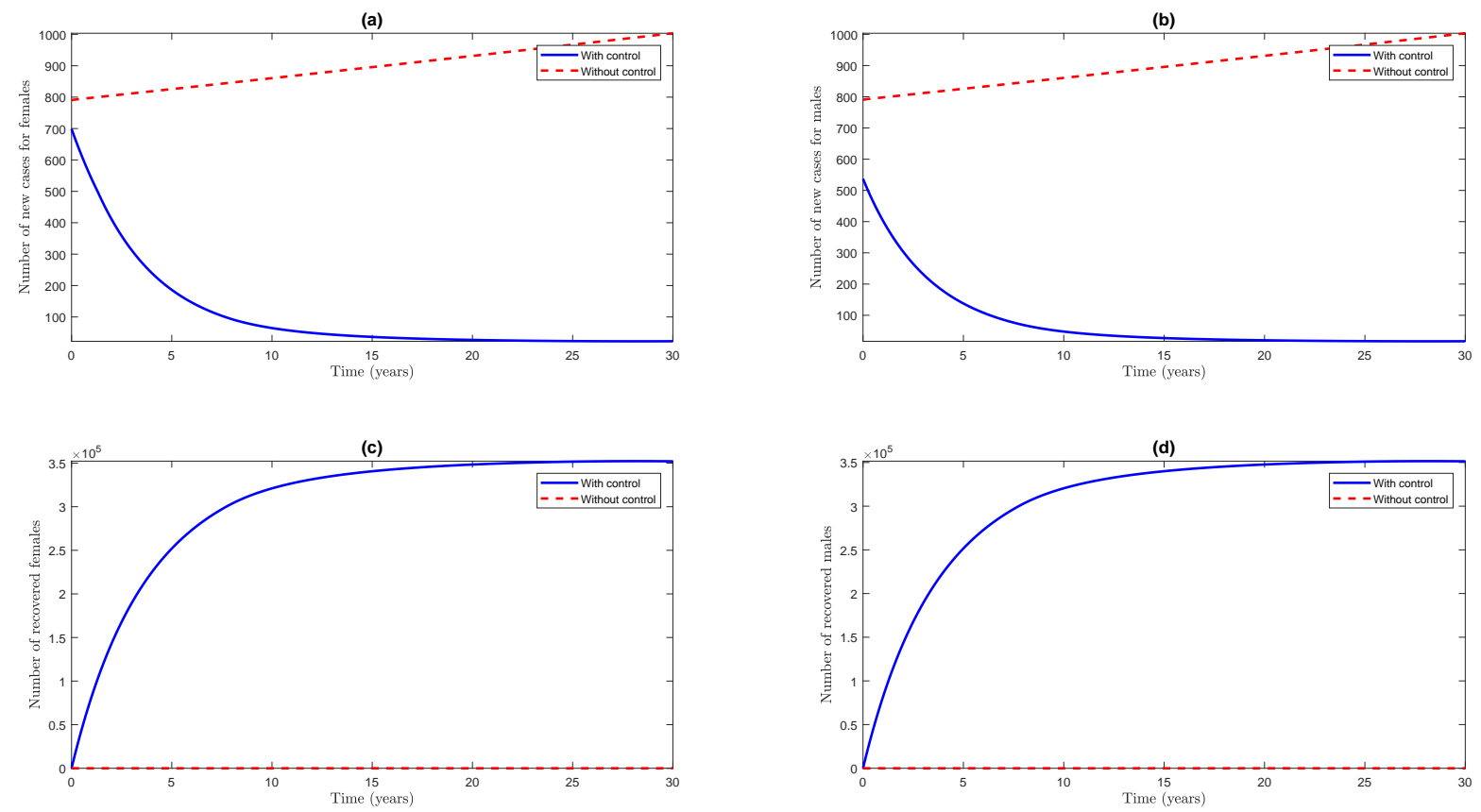

Figure 7: Number of (a) new cases for females (b) new cases for males (c) recovered females and (d) recovered males as a function of time in the presence and absence of optimal control. All parameter value used are as given in Table 2 . 


\section{Discussion and Conclusions}

Syphilis is one of the most deadly sexually-transmitted diseases. Every year, it accounts for over 12 million cases and over 200,000 fatalities globally (inflicting major public health burden in both developing and developed of the world) [1, 2, 4, 8, 10, 63]. Control against syphilis include rapid diagnosis of suspected cases, treatment of confirmed cases and condom use.

This study presents a new deterministic model for gaining insight into the transmission dynamics of syphilis in a population. In addition to allowing for the assessment of the population-level impact of treatment and condom use strategies, the model includes some pertinent features of the disease, such as the multiple stages of infection in untreated patients (represented by "early" and "late" stage of infection) [10] and allowing for reinfection of, and disease relapse from, recovered individuals.

Rigorous qualitative analysis of the model shows that it undergoes the phenomenon of backward bifurcation when the associated reproduction number of the model (denoted by $\mathcal{R}_{0}$ ) is less than unity. The epidemiological consequence of backward bifurcation, a dynamic phenomenon characterised by the co-existence of the stable disease-free equilibrium and a stable endemic equilibrium when $\mathcal{R}_{0}$ is less than unity, is that the classical epidemiological requirement of bringing (and maintaining) $\mathcal{R}_{0}$ to a value less than unity, while necessary, is no longer sufficient for the effective control (or elimination) of the disease $[10,14,20,26]$. In a backward bifurcation situation, effective disease control is dependent on the initial sizes of the sub-populations of the model. In other words, the presence of backward bifurcation in the transmission dynamics of a disease makes its effective control difficult. It is shown that the backward bifurcation phenomenon of the model is caused by the reinfection of recovered individuals.

It is worth emphasizing that, although the reinfection of recovered individuals is an important and well-known feature of syphilis transmission dynamics [32], many of the published modeling studies in the literature do not incorporate such property [18, 38, 28, 20, 29] (and, as a consequence, fail to capture the reinfection-induced backward bifurcation property of the disease). This study is one of the very first to theoretically illustrate the phenomenon of backward bifurcation in syphilis transmission dynamics (Milner and Zhao [25] also showed the presence of backward bifurcation in syphilis dynamics, and used this property to explain the resurgence of syphilis in recent decades). It is shown that, in the absence of backward bifurcation (i.e., when reinfection of recovered individuals does not occur), the disease-free equilibrium of the model is globally asymptotically stable whenever the associated reproduction number is less than unity. Thus, for the case when reinfection of recovered individuals is small enough, the im-

plementation of the two control strategies adopted (condom use and treatment of confirmed cases) can lead to the effective control (or elimination) of the disease from the community if they can 
result in bringing (and maintaining) the associated reproduction number of the model to a value less than unity. Thus, this study suggests that the prospects of the effective control of syphilis in a population, using treatment and condom strategies, are promising.

The model developed in this study consists of numerous parameters, and uncertainties were expected to exist in the estimate of their values used in the numerical simulations of the model. The effect of such uncertainties (on the simulation results obtained) was assessed using Latin Hypercube Sampling [60], with the reproduction number of the model as the response function. It is shown, using data relevant to the transmission dynamics of the model in Nigeria [11], that the reproduction number of the model lie within the range $(3.19,6.15)$, with a mean of 4.44 . Thus, this study suggests that the current level of treatment and condom strategies are inadequate to effectively contain the spread of the disease in the country (since they fail to bring the mean value of $\mathcal{R}_{0}$ to a value less than unity). In other words, more needs to be done in terms of increasing compliance in condom use, rapid diagnosis and treatment of confirmed cases and public health education and counselling to encourage safer sex practices.

Detailed sensitivity analysis of the model shows that the most important parameters that affect the reproduction number of the model (hence, disease burden) are the condom efficacy, condom compliance, proportion of treated individuals in the early stage who recovered and the modification parameter associated with the decrease in recovery rate for individuals who failed treatment. This suggests (as expected) that increasing condom compliance and recovery rate of those who failed treatment will decrease syphilis burden in the community.

Optimal control analysis, using Pontraygin's Maximum Principle, was carried out to assess the impact of the cost of implementing the controls (singular and combined administration of condom use and treatment strategies). The simulation results obtained show that, when the cost of implementing the controls is low, treatment-only strategy is significantly more effective than the condom-use only strategy. In particular, while the treatment-only strategy accounted for 642,360 new cases over a 30-day period, the condom-only strategy resulted in 1.3 million cases over the same time period. In other words, these simulation suggests that, in this scenario (with low cost of implementation), the control resources should be targeted towards encouraging/enhancing treatment use in the community. The combined condom-treatment strategy is, expectedly, shown to be more effective than the treatment-only strategy (it accounted for new cases over the 30-day period). However, for the case when the cost of implementation of the controls is high, the three strategies are equally as ineffective (each resulting in nearly 2 million new cases over 30 days). Thus, this study suggests emphasizing treatment ahead of condom use if the cost of implementation of controls is low. This study is perhaps the first to apply optimal control to syphilis transmission dynamics (the models in $[18,38,28,20,29]$ do not include such analysis). Overall, this study 
shows that effective control of syphilis in a population, using condom and treatment strategies, is feasible if their effectiveness level (especially condom compliance rate) are high enough.

The study is preliminary, and can be extended in numerous ways, such as including the effect of vertical transmission, testing of pregnant women and their sexual partners and public health education and counselling for safer sex practices. Moreover, the study can be extended to include the effect of age structure and account for all four stages of syphilis disease. Further theoretical results, such as the global asymptotic stability of the unique endemic equilibrium (which exists for $\tilde{\mathcal{R}}_{0}>1$ in the absence of reinfection), can also be explored.

\section{Acknowledgements}

Two of the authors (YAT and JMSL) are grateful to the DST/NRF SARChI Chair in Mathematical Models and Methods in Bioengineering and Biosciences for generous financial support. The authors are grateful to the anonymous reviewers for their constructive comments, which have improved the manuscript.

\section{Appendix A: Proof of Theorem 2.1}

Proof. It is easy to check that the equations for male and female susceptible individuals in the model (2.3) lead to the following first-order inequalitity equations:

$$
\frac{d S_{F}}{d t}+\left(\lambda_{F}+\mu\right) S_{F}>0 \text { and } \frac{d S_{M}}{d t}+\left(\lambda_{M}+\mu\right) S_{M}>0
$$

Multiplying these inequalities by the integrating factors $\rho_{F}(t)=\exp \left(\int_{0}^{t}\left(\lambda_{F}(s)+\mu\right) d s\right.$ and $\rho_{M}(t)=$ $\exp \left(\int_{0}^{t}\left(\lambda_{M}(s)+\mu\right) d s\right.$ and observing that

$$
\rho_{F}\left[\frac{d S_{F}}{d t}+\left(\lambda_{F}+\mu\right) S_{F}\right]=\frac{d\left(S_{F} \rho_{F}\right)}{d t} \text { and } \rho_{F}\left[\frac{d S_{F}}{d t}+\left(\lambda_{M}+\mu\right) S_{M}\right]=\frac{d\left(S_{M} \rho_{M}\right)}{d t}
$$

then integration with respect to time from 0 to $t$ gives $S_{F}(t) \geq 0$ and $S_{M}(t) \geq 0$ at all times, respectively. However, this direct approach does not apply to the remaining equations. Nevertheless, having the nonnegativity of $S_{F}$ and $S_{M}$ in mind, it can be shown that the remaining eight equations in the model (2.3) form a monotone system. Consequently, all its solutions corresponding to positive initial data remain positive at all times $t \geq 0$ (see [64]).

By adding the first five and the last five equations of the model (2.3), we obtain the conservation 
law

$$
\begin{aligned}
& \frac{d N_{F}}{d t}=\Lambda_{F}-\mu N_{F}-\delta_{F} I_{F L} \leq \Lambda_{F}-\mu N_{F}, \\
& \frac{d N_{M}}{d t}=\Lambda_{M}-\mu N_{M}-\delta_{M} I_{M L} \leq \Lambda_{M}-\mu N_{M} .
\end{aligned}
$$

Thus, a standard comparison theorem or Gronwall inequality can be used to show that the general a priori estimates below hold.

$$
\begin{aligned}
& 0 \leq N_{F}(t) \leq N_{F}(0) e^{-\mu t}+\frac{\Lambda_{F}}{\mu}\left(1-e^{-\mu t}\right) \\
& 0 \leq N_{M}(t) \leq N_{M}(0) e^{-\mu t}+\frac{\Lambda_{M}}{\mu}\left(1-e^{-\mu t}\right) .
\end{aligned}
$$

In particular, we have a priori estimates

$$
\begin{aligned}
& 0 \leq N_{F}(t) \leq \frac{\Lambda_{F}}{\mu} \text { if } N_{F}(0) \leq \frac{\Lambda_{F}}{\mu} \\
& 0 \leq N_{M}(t) \leq \frac{\Lambda_{M}}{\mu} \text { if } N_{M}(0) \leq \frac{\Lambda_{M}}{\mu}
\end{aligned}
$$

Combining these a priori estimates and the fact that the right-hand side of the model (2.3) is locally Lipschitz, we conclude that there exists a unique global solution in the domain $\Omega$ (see Theorem 2.1.5 in [33]). Thus, the model (2.3) is a dynamical system on $\Omega$. On the other hand, if a solution is outside the region $\Omega$, that is

$$
N_{F}(t) \geq \frac{\Lambda_{F}}{\mu} \text { and } N_{M}(t) \geq \frac{\Lambda_{M}}{\mu}
$$

then, it follows from the above conservation law that $\frac{d N_{F}}{d t} \leq 0$ and $\frac{d N_{M}}{d t} \leq 0$. Hence, the above general a priori estimates show that $N_{F}(t)$ tends to $\frac{\Lambda_{F}}{\mu}$ and $N_{M}(t)$ tends $\frac{\Lambda_{M}}{\mu}$ as $t \rightarrow \infty$. Thus, the region $\Omega$ is attracting.

\section{Appendix B: Proof of Theorem 3.3}

Proof. It is convenient to define the following change of variables $x_{1}=S_{F}, x_{2}=I_{F E}, x_{3}=$ $I_{F L}, x_{4}=Q_{F}, x_{5}=R_{F}, x_{6}=S_{M}, x_{7}=I_{M E}, x_{8}=I_{M L}, x_{9}=Q_{F}$ and $x_{10}=R_{M}$, so that $N_{f}=x_{1}+x_{2}+x_{3}+x_{4}+x_{5}$ and $N_{m}=x_{6}+x_{7}+x_{8}+x_{9}+x_{10}$. Furthermore, by using the vector notation $X=\left(x_{1}, x_{2}, x_{3}, x_{4}, x_{5}, x_{6}, x_{7}, x_{8}, x_{9}, x_{10}\right)^{T}$, the model (2.3) can be written in the 
form $\frac{d X}{d t}=F=\left(f_{1}, f_{2}, f_{3}, f_{4}, f_{5}, f_{6}, f_{7}, f_{8}, f_{9}, f_{10}\right)^{T}$, as follows:

$$
\begin{aligned}
\frac{d x_{1}}{d t} & =\Lambda_{F}-\left(\lambda_{F}+\mu\right) x_{1} \\
\frac{d x_{2}}{d t} & =\lambda_{F}\left(x_{1}+\theta_{F} x_{5}\right)+\gamma_{F} x_{5}-k_{1} x_{1} \\
\frac{d x_{3}}{d t} & =\sigma_{F} x_{2}-k_{2} x_{3}, \\
\frac{d x_{4}}{d t} & =\left(1-f_{F}\right) \tau_{F} x_{2}+\left(1-g_{F}\right) \alpha_{T} \tau_{F} x_{3}-k_{3} x_{4}, \\
\frac{d x_{5}}{d t} & =f_{F} \tau_{F} x_{2}+g_{F} \alpha_{T} \tau_{F} x_{3}+\eta_{T} \tau_{F} x_{4}-\theta_{F} \lambda_{F} x_{5}-k_{4} x_{5}, \\
\frac{d x_{6}}{d t} & =\Lambda_{M}-\left(\lambda_{M}+\mu\right) x_{6}, \\
\frac{d x_{7}}{d t} & =\lambda_{M}\left(x_{6}+\theta_{M} x_{10}\right)+\gamma_{M} x_{10}-k_{5} x_{7}, \\
\frac{d x_{8}}{d t} & =\sigma_{M} x_{7}-k_{6} x_{8} \\
\frac{d x_{9}}{d t} & =\left(1-f_{M}\right) \tau_{M} x_{7}+\left(1-g_{M}\right) \alpha_{T} \tau_{M} x_{8}-k_{7} x_{9}, \\
\frac{d x_{10}}{d t} & =f_{M} \tau_{M} x_{7}+g_{M} \alpha_{T} \tau_{M} x_{8}+\eta_{T} \tau_{M} x_{9}-\theta_{M} \lambda_{M} x_{10}-k_{8} x_{10},
\end{aligned}
$$

where (as before),

$$
\begin{aligned}
& k_{1}=\sigma_{F}+\tau_{F}+\mu, \quad k_{2}=\alpha_{T} \tau_{F}+\delta_{F}+\mu, \quad k_{3}=\eta_{T} \tau_{F}+\mu, \quad k_{4}=\mu+\gamma_{F}, \\
& k_{5}=\sigma_{M}+\tau_{M}+\mu, \quad k_{6}=\alpha_{T} \tau_{M}+\delta_{M}+\mu, \quad k_{7}=\eta_{T} \tau_{M}+\mu, \quad k_{8}=\mu+\gamma_{M},
\end{aligned}
$$

and, now,

$$
\begin{gathered}
\lambda_{F}=\left(1-\epsilon_{F} c_{F}\right) \frac{p_{F} \beta_{F}}{N_{M}}\left(x_{7}+\nu_{M} x_{8}+\xi_{M} x_{9}\right), \\
\lambda_{M}=\left(1-\epsilon_{M} c_{M}\right) \frac{p_{M} \beta_{M}}{N_{F}}\left(x_{2}+\nu_{F} x_{3}+\xi_{F} x_{4}\right) .
\end{gathered}
$$

The Jacobian of the system (B.1), at the associated DFE $\left(\mathcal{E}_{0}\right)$, is given by

$$
J\left(\mathcal{E}_{0}\right)=\left(\begin{array}{ll}
A_{5 \times 5} & B_{5 \times 5} \\
C_{5 \times 5} & D_{5 \times 5}
\end{array}\right),
$$

where, 


$$
\begin{aligned}
& A=\left(\begin{array}{ccccc}
-\mu & 0 & 0 & 0 & 0 \\
0 & -k_{1} & 0 & 0 & \gamma_{F} \\
0 & \sigma_{F} & -k_{2} & 0 & 0 \\
0 & \left(1-f_{F}\right) \tau_{F} & \left(1-g_{F}\right) \alpha_{T} \tau_{F} & -k_{3} & 0 \\
0 & \tau_{F} f_{F} & \alpha_{T} \tau_{F} g_{F} & \eta_{T} \tau_{F} & -k_{4}
\end{array}\right), B=\left(\begin{array}{ccccc}
0 & -x_{F} & -x_{F} \nu_{M} & -x_{F} \xi_{M} & 0 \\
0 & x_{F} & x_{F} \nu_{M} & x_{F} \xi_{M} & 0 \\
0 & 0 & 0 & 0 & 0 \\
0 & 0 & 0 & 0 & 0 \\
0 & 0 & 0 & 0 & 0
\end{array}\right) \\
& C=\left(\begin{array}{ccccc}
0 & -x_{M} & -x_{M} \nu_{F} & -x_{M} \xi_{F} & 0 \\
0 & x_{M} & x_{M} \nu_{F} & x_{M} \xi_{F} & 0 \\
0 & 0 & 0 & 0 & 0 \\
0 & 0 & 0 & 0 & 0 \\
0 & 0 & 0 & 0 & 0
\end{array}\right) \text { and } D=\left(\begin{array}{ccccc}
-\mu & 0 & 0 & 0 & 0 \\
0 & -k_{5} & 0 & 0 & \gamma_{M} \\
0 & \sigma_{M} & -k_{6} & 0 & 0 \\
0 & \left(1-f_{M}\right) \tau_{M} & \left(1-g_{M}\right) \alpha_{T} \tau_{M} & -k_{7} & 0 \\
0 & \tau_{M} f_{M} & \alpha_{T} \tau_{M} g_{M} & \eta_{T} \tau_{F} & -k_{8}
\end{array}\right) \text {, }
\end{aligned}
$$

with,

$$
x_{F}=\frac{\left(1-\epsilon_{F} c_{F}\right) p_{F} \beta_{F} \Lambda_{F}}{\Lambda_{M}}, \quad x_{M}=\frac{\left(1-\epsilon_{M} c_{M}\right) p_{M} \beta_{M} \Lambda_{M}}{\Lambda_{F}} .
$$

Consider, next, the case when $\mathcal{R}_{0}=1$, and choose $p_{M}$ as a bifurcation parameter. Solving for $p_{M}$ from $\mathcal{R}_{0}=1$ gives

$$
p_{M}=p_{M}^{*}=\frac{\left(T_{F 1}+T_{F 2}\right)\left(T_{M 1}+T_{M 2}\right)}{p_{F} \beta_{F} \beta_{M} k_{4} k_{8}\left(1-\epsilon_{M} c_{M}\right)\left(1-\epsilon_{F} c_{F}\right) T_{F} T_{M}},
$$

where (as before),

$$
\begin{aligned}
T_{F} & =\tau_{F} \xi_{F}\left[k_{2}\left(1-f_{F}\right)+\alpha_{T} \sigma_{F}\left(1-g_{F}\right)\right]+k_{3}\left(k_{2}+\nu_{F} \sigma_{F}\right), \\
T_{F 1} & =k_{2}\left\{\mu^{3}+\left[\sigma_{F}+\gamma_{F}+\tau_{F}\left(1+\eta_{T}\right)\right] \mu^{2}+\left[\eta_{T}\left(\tau_{F}+\sigma_{F}+\gamma_{F}\right)+\gamma_{F}\left(1-f_{F}\right)\right] \tau_{F} \mu\right\}, \\
T_{F 2} & =\gamma_{F} \sigma_{F}\left\{\mu^{2}+\left[\delta_{F}+\eta_{T} \tau_{F}+\tau_{F} \alpha_{T}\left(1-g_{F}\right)\right] \mu+\eta_{T} \tau_{F} \delta_{F}\right\}, \\
T_{M} & =\tau_{M} \xi_{M}\left[k_{6}\left(1-f_{M}\right)+\alpha_{T} \sigma_{M}\left(1-g_{M}\right)\right]+k_{7}\left(k_{6}+\nu_{M} \sigma_{M}\right), \\
T_{M 1} & =k_{6}\left\{\mu^{3}+\left[\sigma_{M}+\gamma_{M}+\tau_{M}\left(1+\eta_{T}\right)\right] \mu^{2}+\left[\eta_{T}\left(\tau_{M}+\sigma_{M}+\gamma_{M}\right)+\gamma_{M}\left(1-f_{M}\right)\right] \tau_{M} \mu\right\}, \\
T_{M 2} & =\gamma_{M} \sigma_{M}\left\{\mu^{2}+\left[\delta_{M}+\eta_{T} \tau_{M}+\tau_{M} \alpha_{T}\left(1-g_{M}\right)\right] \mu+\eta_{T} \tau_{M} \delta_{M}\right\} .
\end{aligned}
$$

It can be verified that the transformed system (B.1), with $p_{M}=p_{M}^{*}$, has a non hyperbolic equilibrium point such that the linearized system has a simple eigenvalue with zero real part, and all other eigenvalues have negative real parts. Hence, the centre manifold theory $[13,15,34]$ can be used to analyse the dynamics of the model (B.1) near $p_{M}=p_{M}^{*}$. Using the notation in [15], the following computations are carried out. 


\section{Eigenvectors of $\left.J\left(\mathcal{E}_{0}\right)\right|_{p_{M}=p_{M}^{*}}$}

It can be shown that the Jacobian, $J\left(\mathcal{E}_{0}\right)$ at $p_{M}=p_{M}^{*}$ (denoted by $J_{p_{M}^{*}}$ ) has a right eigenvector (associated with the zero eigenvalue), given by

$$
\mathbf{w}=\left[w_{1}, w_{2}, w_{3}, w_{4}, w_{5}, w_{6}, w_{7}, w_{8}, w_{9}, w_{10}\right]^{T}
$$

where,

$$
\begin{aligned}
w_{1} & =-\frac{p_{F} \beta_{F} \Lambda_{F} w_{7}\left(1-\epsilon_{F} c_{F}\right)\left[\alpha_{T} \sigma_{M} \tau_{M} \xi_{M}\left(1-g_{M}\right)+\tau_{M} \xi_{M} k_{6}\left(1-f_{M}\right)+k_{7}\left(k_{6}+\nu_{M} \sigma_{M}\right)\right]}{\mu \Lambda_{M} k_{6} k_{7}}, \\
w_{2} & =\frac{p_{F} \beta_{F} \Lambda_{F} k_{2} k_{3} k_{4} w_{7}\left(1-\epsilon_{F} c_{F}\right)\left[\alpha_{T} \sigma_{M} \tau_{M} \xi_{M}\left(1-g_{M}\right)+\tau_{M} \xi_{M} k_{6}\left(1-f_{M}\right)+k_{7}\left(k_{6}+\nu_{M} \sigma_{M}\right)\right]}{\Lambda_{M} k_{6} k_{7} T_{F 1}}, \quad w_{5}=\frac{f_{F} \tau_{F} w_{2}+g_{F} \alpha_{T} \tau_{F} w_{3}+\eta_{T} \tau_{F} w_{4}}{k_{4}}, \\
w_{3}= & \frac{\sigma_{F} w_{2}}{k_{2}}, w_{4}=\frac{\left(1-f_{F}\right) \tau_{F} w_{2}+\left(1-g_{F}\right) \alpha_{T} \tau_{F} w_{3}}{k_{4}}, \quad w_{7}=\frac{\sigma_{M} w_{7}}{k_{6}}, \\
w_{6}= & -\left(1-\epsilon_{M} c_{M}\right) p_{M} \beta_{F}\left(w_{2}+\nu_{F} w_{3}+\xi_{F} w_{4}\right), \quad w_{7}, w_{10}=\frac{f_{M} \tau_{M} w_{7}+g_{M} \alpha_{T} \tau_{M} w_{8}+\eta_{T} \tau_{M} w_{9}}{k_{8}} .
\end{aligned}
$$

Furthermore, $J_{p_{M}^{*}}$ has a left eigenvector (associated with the zero eigenvalue), given by

$$
\mathbf{v}=\left[v_{1}, v_{2}, v_{3}, v_{4}, v_{5}, v_{6}, v_{7}, v_{8}, v_{9}, v_{10}\right]
$$

with,

$$
\begin{gathered}
v_{1}=0 \\
v_{2}=\frac{p_{M} \beta_{F} k_{4} v_{7}\left(1-\epsilon_{M} c_{M}\right)\left[\alpha_{T} \sigma_{F} \tau_{F} \xi_{F}\left(1-g_{F}\right)+\tau_{F} \xi_{F} k_{2}\left(1-f_{F}\right)+k_{3}\left(k_{2}+\nu_{F} \sigma_{F}\right)\right]}{T_{F 1}}, \\
v_{3}=\frac{\left(1-g_{F}\right) \alpha_{T} \tau_{F} v_{4}}{k_{2}}+\frac{g_{F} \alpha_{T} \tau_{F} \gamma_{F} v_{2}}{k_{2} k_{4}}+\frac{p_{M} \beta_{F} \nu_{F}\left(1-c_{M} \epsilon_{M}\right) v_{7}}{k_{2}}, \\
v_{4}=\frac{p_{M} \beta_{F} v_{7}\left(1-\epsilon_{M} c_{M}\right)\left\{\eta_{T} \gamma_{F} \tau_{F}\left(k_{2}+\nu_{F} \sigma_{F}\right)+\xi_{F}\left[k_{1} k_{2} k_{4}-\gamma_{F} \tau_{F}\left(f_{F} k_{2}+g_{F} \alpha_{T} \sigma_{F}\right)\right]\right\}}{T_{F 1}}, \\
v_{5}=\frac{\gamma_{F} v_{2}}{k_{4}}, \quad v_{6}=0, \quad v_{7}=v_{7}>0, \\
v_{8}=\frac{p_{F} \beta_{F}\left(1-c_{F} \epsilon_{F}\right) \nu_{M} \Lambda_{F} v_{2}}{\Lambda_{M} k_{6}}+\frac{\left(1-g_{M}\right) \alpha_{T} \tau_{M} v_{9}}{k_{6}}+\frac{g_{M} \alpha_{T} \tau_{M} \gamma_{M} v_{7}}{k_{6} k_{8}} \\
v_{9}=\frac{p_{F} \beta_{F}\left(1-c_{F} \epsilon_{F}\right) \xi_{M} \Lambda_{F} v_{2}}{\Lambda_{M} k_{7}}+\frac{\eta_{T} \tau_{M} \gamma_{M} v_{7}}{k_{7} k_{8}}, \quad v_{10}=\frac{\gamma_{M} v_{7}}{k_{8}} .
\end{gathered}
$$




\section{Computations of bifurcation coefficients $a$ and $b$}

It can be shown, by computing the non-zero partial derivatives of $F$ at the DFE $\left(\mathcal{E}_{0}\right)$ and simplifying, that

$$
\begin{aligned}
a= & \sum_{k, i, j=1}^{10} v_{k} w_{i} w_{j} \frac{\partial^{2} f_{k}}{\partial x_{i} \partial x_{j}}\left(\mathcal{E}_{0}\right) \\
= & \frac{2 \mu}{\Lambda_{F}}\left(1-\epsilon_{F} c_{F}\right) p_{F} \beta_{M}\left[\theta_{F}\left(v_{2}-v_{5}\right) w_{5}-v_{2} \sum_{i=2}^{5} w_{i}\right]\left(w_{7}+\nu_{M} w_{8}+\xi_{M} w_{9}\right) \\
& +\frac{2 \mu}{\Lambda_{M}}\left(1-\epsilon_{M} c_{M}\right) p_{M} \beta_{F}\left[\theta_{M}\left(v_{7}-v_{10}\right) w_{10}-v_{7} \sum_{i=7}^{10} w_{i}\right]\left(w_{2}+\nu_{F} w_{3}+\xi_{F} w_{4}\right) .
\end{aligned}
$$

Thus, the bifurcation coefficient, $a$, is positive whenever

$$
\theta_{F}\left(v_{2}-v_{5}\right) w_{5}-v_{2} \sum_{i=2}^{5} w_{i}>0 \text { and } \theta_{M}\left(v_{7}-v_{10}\right) w_{10}-v_{7} \sum_{i=7}^{10} w_{i}>0 .
$$

Furthermore, it can be shown that:

$$
\begin{aligned}
b & =\sum_{k, i=1}^{10} v_{k} w_{i} \frac{\partial^{2} f_{k}}{\partial x_{i} \partial p_{M}^{*}}\left(\mathcal{E}_{0}\right), \\
& =\beta_{F} v_{7}\left(1-\epsilon_{M} c_{M}\right)\left(w_{2}+\nu_{F} w_{3}+\xi_{F} w_{4}\right)>0 .
\end{aligned}
$$

Hence, it follows, from Theorem 4.1 in [15], that the model (2.3) exhibits a backward bifurcation at $\mathcal{R}_{0}=1$ whenever

$$
\theta_{F}\left(v_{2}-v_{5}\right) w_{5}-v_{2} \sum_{i=2}^{5} w_{i}>0 \text { and } \theta_{M}\left(v_{7}-v_{10}\right) w_{10}-v_{7} \sum_{i=7}^{10} w_{i}>0 .
$$




\section{Appendix C: Proof of Theorem 3.4}

Proof. Setting $\theta_{F}=\theta_{M}=0$ in the expression for the bifurcation coefficient $a$, given in (B.3) above, gives (it should be noted that the eigenvectors $v_{2}, v_{7}, w_{2}, w_{3}, w_{4}, w_{5}, w_{7}, w_{8}, w_{9}, w_{10}$ are all positive)

$$
\begin{aligned}
a= & \sum_{k, i, j=1}^{10} v_{k} w_{i} w_{j} \frac{\partial^{2} f_{k}}{\partial x_{i} \partial x_{j}}\left(\mathcal{E}_{0}\right), \\
= & -\left[\frac{2 \mu}{\Lambda_{F}}\left(1-\epsilon_{F} c_{F}\right) p_{F} \beta_{M} v_{2}\left(w_{7}+\nu_{M} w_{8}+\xi_{M} w_{9}\right) \sum_{i=2}^{5} w_{i}\right. \\
& \left.+\frac{2 \mu}{\Lambda_{M}}\left(1-\epsilon_{M} c_{M}\right) p_{M} \beta_{F} v_{7}\left(w_{2}+\nu_{F} w_{3}+\xi_{F} w_{4}\right) \sum_{i=7}^{10} w_{i}\right]<0 .
\end{aligned}
$$

Thus, it follows from Theorem 4.1 in [15] that the model (2.3) with $\theta_{F}=\theta_{M}=0$ will not undergo a backward bifurcation at $\tilde{\mathcal{R}}_{0}=1$.

\section{Appendix D: Proof of Theorem 3.5}

Proof. It is worth noting, first of all, that the equations for the infected components of the model (2.3) with $\theta_{F}=\theta_{M}=0$ can be written in matrix-vector form:

$$
\frac{d X(t)}{d t}=\left[(F-V)-\left(1-\frac{S_{F}}{N_{F}}\right) K_{1}-\left(1-\frac{S_{M}}{N_{M}}\right) K_{2}\right] X(t)
$$

where $X(t)=\left(I_{F E}(t), I_{F L}(t), Q_{F}(t), R_{F}(t), I_{M E}(t), I_{M L}(t), Q_{M}(t), R_{M}(t)\right)^{T}$ and the matrices $F$ and $V$ are given in Section 3, Furthermore,

$$
K_{1}=\left(\begin{array}{cccccccc}
0 & 0 & 0 & 0 & x_{F} & x_{F} \nu_{M} & x_{F} \xi_{M} & 0 \\
0 & 0 & 0 & 0 & 0 & 0 & 0 & 0 \\
0 & 0 & 0 & 0 & 0 & 0 & 0 & 0 \\
0 & 0 & 0 & 0 & 0 & 0 & 0 & 0 \\
0 & 0 & 0 & 0 & 0 & 0 & 0 & 0 \\
0 & 0 & 0 & 0 & 0 & 0 & 0 & 0 \\
0 & 0 & 0 & 0 & 0 & 0 & 0 & 0 \\
0 & 0 & 0 & 0 & 0 & 0 & 0 & 0
\end{array}\right) \text { and } K_{2}=\left(\begin{array}{cccccccc}
0 & 0 & 0 & 0 & 0 & 0 & 0 & 0 \\
0 & 0 & 0 & 0 & 0 & 0 & 0 & 0 \\
0 & 0 & 0 & 0 & 0 & 0 & 0 & 0 \\
0 & 0 & 0 & 0 & 0 & 0 & 0 & 0 \\
x_{M} & x_{M} \nu_{F} & x_{M} \xi_{F} & 0 & 0 & 0 & 0 & 0 \\
0 & 0 & 0 & 0 & 0 & 0 & 0 & 0 \\
0 & 0 & 0 & 0 & 0 & 0 & 0 & 0 \\
0 & 0 & 0 & 0 & 0 & 0 & 0 & 0
\end{array}\right) .
$$


Since $K_{1}$ and $K_{2}$ are non-negative matrices and $S_{F}(t) \leq N_{M}(t)$ and $S_{M}(t) \leq N_{M}(t)$ in $\Omega$, it follows that

$$
\frac{d X(t)}{d t} \leq(F-V) X(t)
$$

Using the fact that the eigenvalues of the matrix $F-V$ all have negative real parts (i.e., $\rho\left(F V^{-1}\right)<$ 1 if $\tilde{\mathcal{R}}_{0}<1$ ), it follows that the linearized differential inequality system (2.3) is stable whenever $\tilde{\mathcal{R}}_{0}<1$. Thus, by Comparison Theorem [22],

$$
\lim _{t \rightarrow \infty}\left(I_{F E}(t), I_{F L}(t), Q_{F}(t), R_{F}(t), I_{M E}(t), I_{M L}(t), Q_{M}(t), R_{M}(t)\right)=(0,0,0,0,0,0,0,0) .
$$

Substituting (D.3) into (2.3), it can be shown that $S_{F}(t) \rightarrow \frac{\Lambda_{F}}{\mu}$ and $S_{M}(t) \rightarrow \frac{\Lambda_{M}}{\mu}$, as $t \rightarrow \infty$. Hence,

$$
\begin{aligned}
& \lim _{t \rightarrow \infty}\left(S_{F}(t), I_{F E}(t), I_{F L}(t), Q_{F}(t), R_{F}(t), S_{M}(t), I_{M E}(t), I_{M L}(t), Q_{M}(t), R_{M}(t)\right) \\
& =\left(\frac{\Lambda_{F}}{\mu}, 0,0,0,0, \frac{\Lambda_{M}}{\mu}, 0,0,0,0\right)=\mathcal{E}_{0} .
\end{aligned}
$$

Thus, every solution to the equations of the model (2.3), with $\theta_{F}=\theta_{M}=0$ and initial conditions in $\Omega$, approaches the $\operatorname{DFE}\left(\mathcal{E}_{0}\right)$ as $t \rightarrow \infty$ whenever $\tilde{\mathcal{R}}_{0}<1$.

\section{Appendix E: Proof of Theorem 5.1}

Proof. Corollary 4.1 of [52] gives the existence of an optimal control quadruple $\left(\tau_{F}(t), \tau_{M}(t), c_{F}(t)\right.$, $\left.c_{M}(t)\right)$ due to the convexity of the integrand of $J$ with respect to $\left(\tau_{F}(t), \tau_{M}(t), c_{F}(t), c_{M}(t)\right)$, a priori boundedness of the state solutions, and the local Lipschitz property of the model (2.3) with respect to the state variables. Applying the Pontryagin's Maximum Principle [53], gives

$$
\begin{gathered}
\frac{d \lambda_{S_{F}}}{d t}=-\frac{\partial H}{\partial S_{F}}, \quad \lambda_{S_{F}}\left(t_{f}\right)=0, \quad \frac{d \lambda_{I_{F E}}}{d t}=-\frac{\partial H}{\partial I_{F E}}, \quad \lambda_{I_{F E}}\left(t_{f}\right)=0, \\
\frac{d \lambda_{I_{F L}}}{d t}=-\frac{\partial H}{\partial I_{F L}}, \quad \lambda_{I_{F L}}\left(t_{f}\right)=0, \quad \frac{d \lambda_{Q_{F}}}{d t}=-\frac{\partial H}{\partial Q_{F}}, \quad \lambda_{Q_{F}}\left(t_{f}\right)=0, \\
\frac{d \lambda_{R_{F}}}{d t}=-\frac{\partial H}{\partial R_{F}}, \quad \lambda_{R_{F}}\left(t_{f}\right)=0, \quad \frac{d \lambda_{S_{M}}}{d t}=-\frac{\partial H}{\partial S_{M}}, \quad \lambda_{S_{M}}\left(t_{f}\right)=0, \\
\frac{d \lambda_{I_{M E}}}{d t}=-\frac{\partial H}{\partial I_{M E}}, \quad \lambda_{I_{M E}}\left(t_{f}\right)=0, \quad \frac{d \lambda_{I_{M L}}}{d t}=-\frac{\partial H}{\partial I_{M L}}, \quad \lambda_{I_{M L}}\left(t_{f}\right)=0, \\
\frac{d \lambda_{Q_{M}}}{d t}=-\frac{\partial H}{\partial Q_{M}}, \quad \lambda_{Q_{M}}\left(t_{f}\right)=0, \quad \frac{d \lambda_{R_{M}}}{d t}=-\frac{\partial H}{\partial R_{M}}, \quad \lambda_{R_{M}}\left(t_{f}\right)=0 .
\end{gathered}
$$


Evaluating (E.1) at the optimal control quadruple $\left(\tau_{F}(t), \tau_{M}(t), c_{F}(t), c_{M}(t)\right)$ and the corresponding state variables results in the stated adjoint system (5.4) and (5.5). In the interior of the control set $W$, where $a_{11}<\tau_{F}(t)<a_{12}, a_{21}<\tau_{M}(t)<a_{22}, b_{11}<c_{F}(t)<b_{12}, b_{21}<c_{M}(t)<b_{22}$, the optimality conditions give

$$
\begin{aligned}
\frac{\partial H}{\partial \tau_{F}} & =\omega_{9} \tau_{F}-I_{F E}\left[\lambda_{I_{F E}}-\lambda_{Q_{F}}+f_{F}\left(\lambda_{Q_{F}}-\lambda_{R_{F}}\right)\right]-\alpha_{T} I_{F L}\left(\lambda_{I_{F L}}-\lambda_{Q_{F}}\right) \\
& -\left(\alpha_{T} I_{F L} g_{F}+\eta_{T} Q_{F}\right)\left(\lambda_{Q_{F}}-\lambda_{R_{F}}\right)=0 \\
\frac{\partial H}{\partial \tau_{M}} & =\omega_{10} \tau_{M}-I_{M E}\left[\lambda_{I_{M E}}-\lambda_{Q_{M}}+f_{M}\left(\lambda_{Q_{M}}-\lambda_{R_{M}}\right)\right]-\alpha_{T} I_{M L}\left(\lambda_{I_{M L}}-\lambda_{Q_{M}}\right) \\
& -\left(\alpha_{T} I_{M L} g_{M}+\eta_{T} Q_{M}\right)\left(\lambda_{Q_{M}}-\lambda_{R_{M}}\right)=0 \\
\frac{\partial H}{\partial c_{F}} & =\omega_{11} c_{F}-\frac{\epsilon_{F} p_{F} \beta_{F}\left(I_{M E}+\nu_{M} I_{M L}+\xi_{M} Q_{M}\right)\left[S_{F}\left(\lambda_{I_{F E}}-\lambda_{S_{F}}\right)+\theta_{F} R_{F}\left(\lambda_{I_{F E}}-\lambda_{R_{F}}\right)\right]}{N_{M}}=0 \\
\frac{\partial H}{\partial c_{M}} & =\omega_{12} c_{M}-\frac{\epsilon_{M} p_{M} \beta_{M}\left(I_{F E}+\nu_{F} I_{F L}+\xi_{F} Q_{F}\right)\left[S_{M}\left(\lambda_{I_{M E}}-\lambda_{S_{M}}\right)+\theta_{M} R_{M}\left(\lambda_{I_{M E}}-\lambda_{R_{M}}\right)\right]}{N_{F}}=0 .
\end{aligned}
$$

The optimal control quadruple $\left(\tau_{F}^{*}, \tau_{M}^{*}, c_{F}^{*}, c_{M}^{*}\right)$ can be obtained by solving the optimality conditions above, to obtain $\tau_{F}^{*}=Z_{1}, \tau_{M}^{*}=Z_{2}, c_{F}^{*}=Z_{3}, c_{M}^{*}=Z_{4}$, where, $Z_{1}, Z_{2}, Z_{3}$ and $Z_{4}$ are defined in (5.7). Using the bounds for the control variables in the control set $W$, the controls $\tau_{F}^{*}, \tau_{M}^{*}, c_{F}^{*}$ and $c_{M}^{*}$ can be re-written in the form (5.6), as required.

\section{References}

[1] D. Aadland, D. Finnoff and K. Huang (2013). Syphilis cycles. The B.E. J. Econ. Financ. 13(1): $297-348$.

[2] Z. Chen, G. Zhang, X. Gong, C. Lin, X. Gao, G. Liang, X. Yue, X. Chen and M.S. Cohen (2007). Syphilis in China: results of a national surveillance programme. The Lancet. 369: 132-138.

[3] S.M. Berman, Maternal syphilis: pathophysiology and treatment (2004). Bull. World Health Organ. 82(6):433-438.

[4] L. Doherty, K. Fenton, J. Jones, T. Paine, S. Higgins, D.Williams and A. Palfreeman (2002). Syphilis: old problem, new strategy. BMJ. 325: 153-156.

[5] R. E. LaFond and S. A. Lukehart, Biological basis for syphilis (2006). Clin. Microbiol. Rev. 19: 29-49. 
[6] H. Saloojee, S. Velaphi, Y. Goga, N. Afadapa, R. Steen and O. Lincetto (2004). The prevention and management of congenital syphilis: an overview and recommendations. Bull. World Health Organ. 82: 424-430.

[7] V. Bowen, J. Su, E. Torrone, S. Kidd, and H. Weinstock (2015). increase in incidence of congenital syphilis - United States, 2012-2014. CDC: Morbidity and Mortality Weekly Report. 64(44): 1241-1245. https://www.cdc.gov/mmwr/preview/mmwrhtml/mm6444a3.htm Accessed June $16,2017$.

[8] J. Kiarie, C. K. Mishra, M. Temmerman and L. Newman (2015). Accelerating the dual elimination of mother-to-child transmission of syphilis and HIV: Why now? International Journal of Gynecology and Obstetrics. 130(1): S1-S3.

[9] S. T. Brown, A. Zaidi, S. A. Larsen and G. H. Reynolds (1985). Serological response to syphilis treatment. A new analysis of old data. JAMA. 253(9): 1296-1299.

[10] D. L. Brown and J. E. Frank (2003). Diagnosis and management of syphilis. Am Fam Physician. 68: 283-90.

[11] World Population. WorldoMeters. Website: www.worldometers.info. Accessed 20 May, 2016.

[12] S. Busenberg and K. Cooke (1993). Vertically Transmitted Disease: Models and Dynamics. Springer-Verlag, New York.

[13] J. Carr (1981). Application of Centre of Manifold Theory. Springer-Verlag, New York.

[14] C. Castillo-Chavez, Z. Feng and W. Huang ((2002). On the computation of $\mathcal{R}_{0}$ and its role on global stability. In : C. Castillo-Chavez, P. van den Driessche, D. Kirschner and A. Yakubu (Eds.), in Mathematical Approaches for Emerging and Reemerging Infectious Diseases: An introduction, IMA. Springer, New York, 125: 229-250.

[15] C. Castillo-Chavez and B. Song (2004). Dynamical models of tuberculosis and their application. Mathematical Biosciences and Engineering. 1(2): 361-404.

[16] O. Diekmann and J. Heesterbeek (2000). Mathematical Epidemiology of Infectious Diseases. Wiley.

[17] J. Dushoff, W. Huang and C. Castillo-Chavez (1998). Backward bifurcation and catastrophe in simple models of fatal diseases. Mathematical Biology. 36: 227-248 
[18] G. P. Garnett, S. O. Aral, D. V. Hoyle, W. Cates and R. M. Anderson (1996). The natural history of syphilis implications for the transmission dynamics and control of infection. Sexually Transmitted Disease. 4: 185-200.

[19] A. B. Gumel (2012). Causes of backward bifurcations in some epidemiological models. Mathematical Analysis and Applications. 395: 355-365.

[20] E. Iboi and D. Okuonghae (2016). Population dynamics of a mathematical model for syphilis. Applied Mathematical Modelling. 4: 3573-3590.

[21] C. Kenyon, L. Lynen, E. Florence, S. Caluwaerts, M. Vandenbruaene, L. Apers, P. Soentjens, M. Van Esbroeck and E. Bottieau (2014) Syphilis reinfections pose problems for syphilis diagnosis in Antwerp. Belgium, 1992 to 2012. Euro Surveill. 19(45): pii=20958. Available online: http://www.eurosurveillance.org/ViewArticle. aspx?ArticleId=20958. ..

[22] V. Lakshmikantham, S. Leela and A. Martynyuk (1989). Stability Analysis of Nonlinear Systems. Marcel Dekker, New York, Basel.

[23] L. P. LaSalle (1976). The Stability of Dynamical Systems. Society for Industrial and Applied Mathematics, Philadelphia.

[24] S.A. Larsen, B. M. Steiner and A. H. Rudolph (1995). Laboratory diagnosis and interpretation of tests for syphilis. Clinical Microbiology Reviews. 8(1): 1-21.

[25] F. A. Milner and R. Zhao (2010). A new mathematical model of syphilis. Math. Model. Nat. Phenom. 5(6): 96-108.

[26] S. E. Oliver, M. Aubin, L. Atwell, et al (2016). Ocular syphilis-eight jurisdictions. United States, 2014 - 2015. Morbidity and Mortality Weekly Report. 65:1185-1188. DOI: http://dx.doi.org/10.15585/mmwr.mm6543a2

[27] S. Oswal and G. Lyons (2008). Syphilis in pregnancy. Continuing Education in Anaesthesia Critical Care and Pain. 8(6) 224-227.

[28] G. L. Oxman, K. Smolkowski and J. Noell (1996). Mathematical modelling of epidemic syphilis transmission: implication for syphilis control program. Sexually Transmitting Disease. 23: 3039.

[29] C. M. Saad-Roy, Z. Shuai and P. van den Driessche (2016). A mathematical model of syphilis transmission in an MSM population. Mathematical Biosciences. 277: 59-70. 
[30] O. Sharomi and A. B. Gumel (2009). Re-infection-induced backward bifurcation in the transmission dynamics of Chlamydia trachomatis. Mathematical Analysis and Application. 356: $96-118$.

[31] O. Sharomi, M. A. Safi, A. B. Gumel and D. J. Gerberry (2017). Exogenous re-infection does not always cause backward bifurcation in TB transmission dynamics. Applied Mathematics and Computation. 298: 322-335.

[32] A. G. Schoch and L. J. Alexander (1949). Reinfection and relapse after treatment of early syphilis. Arch Derm Syphilol. 60(5_PART_I): 690-700.

[33] A. M. Stuart and A. R. Humphties (1998). Dynamical Systems and Numerical Analysis. Cambridge University Press, Cambridge.

[34] P. van den Driessche and J. Watmough (2002). Reproduction numbers and sub-threshold endemic equilibria for compartmental models of disease transmission. Mathematical Biosciences 180: $29-48$.

[35] H. Young (1992). Syphilis: new diagnostic directions. International Journal of STD AIDS. 3: 391-413.

[36] H. W. Hethcote (2000). The mathematics of infectious diseases. SIAM Rev., 42(4): 599-653.

[37] O. Diekmann, J. A. P. Heesterbeek and J. A. J. Metz (1990). On the definition and computation of the basic reproduction ratio $\mathcal{R}_{0}$ in models for infectious disease in heterogeneous population. Journal of Mathematical Biology. 28: 365-382.

[38] C. Grassly, C. Fraser and G. P. Garnett (2005). Host immunity and synchronized epidemics of syphilis across the United States. Nature. 433: 117-121.

[39] O. Sharomi, C.N. Podder, A.B. Gumel, E. Elbasha and J. Watmough (2007). Role of incidence function in vaccine-induced backward bifurcation in some HIV models. Mathematical Biosciences. 210(2): 436-463.

[40] T.M. Malik, J. Reimer, A.B. Gumel, E.H. Elbasha and S. Mahmud (2013). The impact of an imperfect vaccine and pap cytology screening on the transmission of human papillomavirus and occurrence of associated cervical dysplasia and cancer. Mathematical Biosciences and Engineering. 10(4): 1173-1205. 
[41] M. Safi and A.B. Gumel (2011). Mathematical analysis of a disease transmission model with quarantine, isolation and an imperfect vaccine. Computers and Mathematics with Applications. 61(10): 3044-3070.

[42] E.H. Elbasha and A.B. Gumel (2006). Theoretical assessment of public health impact of imperfect prophylactic HIV-1 vaccines with therapeutic benefits. Bulletin of Mathematical Biology. 68: 577-614.

[43] O. Sharomi and T. Malik (2017). A model to assess the effect of vaccine compliance on Human Papillomavirus infection and cervical cancer. Applied Mathematical Modelling. 47: 528-550.

[44] F. Nazari, A.B. Gumel and E.H. Elbasha (2015). Differential characteristics of primary infection and reinfection cause backward bifurcation in HCV transmission dynamics. Mathematical Biosciences. 263: 51-69.

[45] S.M. Garba, M.S. Safi and A.B. Gumel (2013). Cross-immunity-induced backward bifurcation for a model of transmission dynamics of two strains of influenza. Nonlinear Analysis. 14(3): 1384-1403.

[46] T. Parran (1938). Syphilis: a public health problem. Science. 87: 147-152.

[47] Chandra N. Podder and Abba B. Gumel (2009). Risk-induced backward bifurcation in HSV-2 transmission dynamics. Dynamics of Continuous, Discrete and Impulsive Systems Series B: Applications and Algorithms. 19: 377-403.

[48] K. Blayneh, A.B. Gumel, S. Lenhart and T. Clayton (2010). Backward bifurcation analysis and optimal control of West Nile virus. Bulletin of Mathematical Biology. 72(4): 1006-1028.

[49] S.M. Garba, A.B. Gumel and M.R. Abu Bakar (2008). Backward bifurcations in dengue transmission dynamics. Mathematical Biosciences. 215(1): 11-25.

[50] A.B. Gumel and B. Song (2008). Existence of multiple-stable equilibria for a multi-drugresistant model of mycobacterium tuberculosis. Mathematical Biosciences and Engineering. 5(3): 437-455.

[51] O. Sharomi and A. B. Gumel (2011). Mathematical study of a risk-structured two-group model for chlamydia transmission dynamics. Applied Mathematical Modelling. 35(8): 3653-3673.

[52] W. H. Fleming and R. W. Rishel (1975). Deterministic and Stochastic Optimal Control. Springer Verlag, New York. 
[53] L. S. Pontryagin, V. G. Boltyanskii, R. V. Gamkrelidze and E. F. Mishchenko (1962). The Mathematical Theory of Optimal Processes. Wiley, New York.

[54] S. Marino, I. B. Hogue, C. J. Ray and D. E. Kirschner (2008). A methodology for performing global uncertainty and sensitivity analysis in systems biology. Journal of theoretical biology. 254(1): 178-196.

[55] D. M. Hamby (1994). A review of techniques for parameter sensitivity analysis of environmental models. Environmental Monitoring and Assessment. 32(2): 135-154.

[56] R. Taylor (1990). Interpretation of the correlation coefficient: a basic review. Journal of Diagnostic Medical Sonography. 6(1): 35-39.

[57] World Bank. Life expectancy at birth. data.worldbank.org. Accessed 12 May, 2016.

[58] G. P. Garnett, S. O. Aral, D. V. Hoyle, W. Cates and R. M. Anderson (1997). The natural history of syphilis: Implications for the transition dynamics and control of infection. Sex. Transm. Dis. 24(4): 185-200.

[59] R. D. Karen and C. W. Susan (1999). The effectiveness of condoms in reducing heterosexual transmission of HIV. Fam. Plan. Persp. 31(6): 272-279.

[60] S. M. Blower and H. Dowlatabadi (1994). Sensitivity and uncertainty analysis of complex models of disease transmission: an HIV model, as an example. Int. Stat. Rev. 2: 229-243.

[61] C. C. R. Downing (1950), Relapse in a Case of Early Syphilis Treated with Penicillin, Br J Vener Dis. 26: 31-32.

[62] M McAsey, L. Mow and W. Han (2012). Convergence of the forward-backward sweep method in optimal control. COmput. Optim. Appl. 53: 207-226.

[63] World Health Report (2004). The world health report 2004 - changing history. http://www . who.int/whr/2004/en/. Assessed June 28, 2017.

[64] H. L. Smith, Monotone dynamical system, an introduction to the theory of competitive and cooperative systems, Math. Surveys and Monographs, Vol. 41, AMS, Providence, Rhode Island, 1995.

[65] A. G. Schoch and L. J. Alexender. Reinfection and relapse after treatment of early syphilis with penicillin. Analysis of One Hundred and Thirty-Seven Cases of "Infectious Failure" in a Total Series of 1,105 Cases. Arch. Derm. Syphilol. 1949;60(5, PART I)(1949): 690-700. 


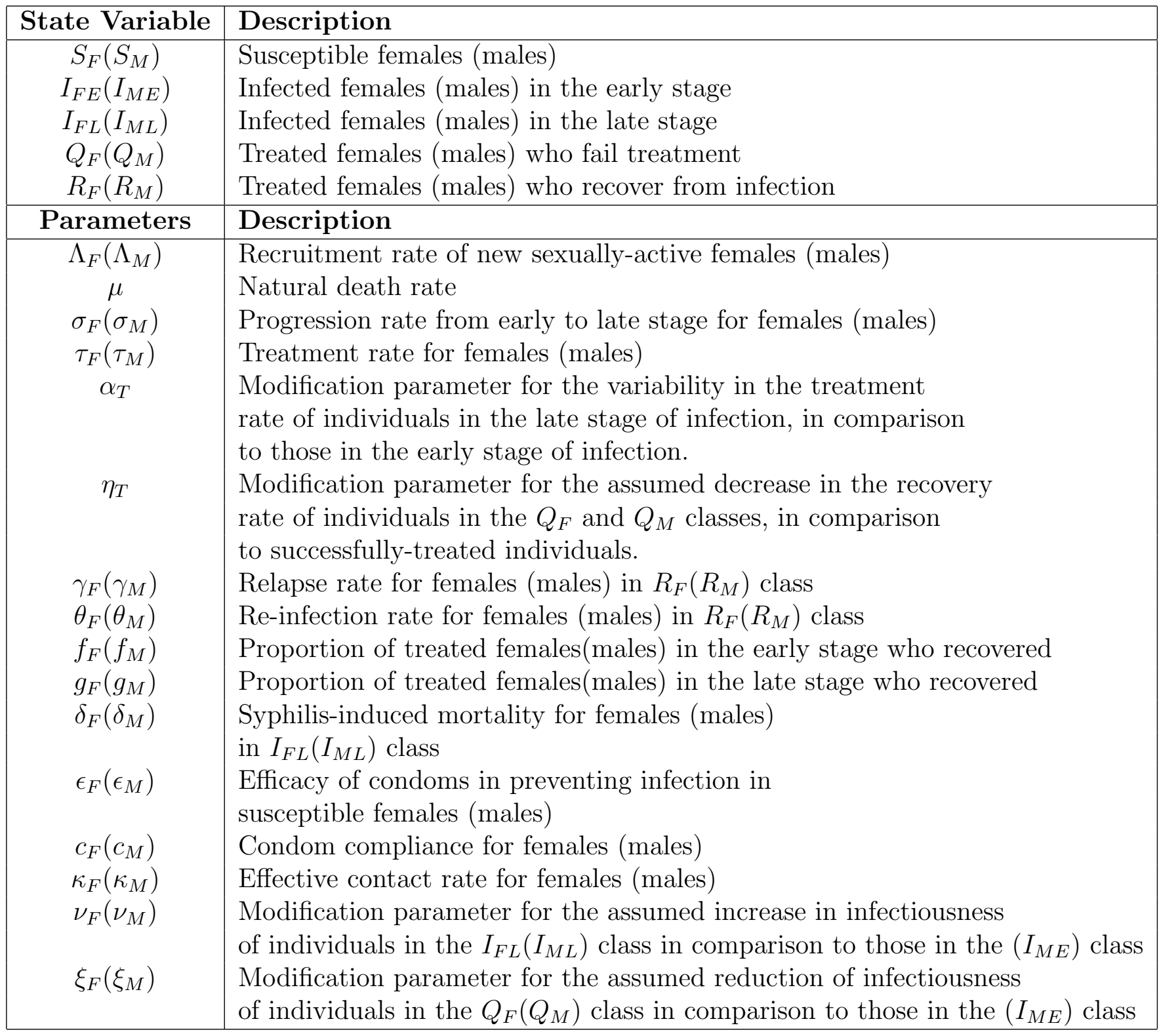

Table 1: Description of state variables and parameters of the model (2.3). 
Table 2: Mean values of the model parameters with their assigned distributions.

\begin{tabular}{l|l|l|l|l}
\hline \hline Parameters & Distribution & Range & Baseline values & References \\
\hline \hline$\mu$ & Normal & $5.08 \times 10^{-5}-5.89 \times 10^{-5}$ & $5.48 \times 10^{-5}$ day $^{-1}$ & {$[57]$} \\
$\sigma_{F}, \sigma_{M}$ & Normal & $3.89 \times 10^{-2}-5.73 \times 10^{-2}$ & $4.762 \times 10^{-2}$ day $^{-1}$ & {$[58]$} \\
$\tau_{F}, \tau_{M}$ & Normal & $2.75 \times 10^{-1}-4.51 \times 10^{-1}$ & $3.6 \times 10^{-1}$ day $^{-1}$ & Implied from [58] \\
$\eta_{T}$ & Normal & $1.99 \times 10^{-2}-1.79 \times 10^{-1}$ & 0.1 & Assumed \\
$\alpha_{T}$ & Normal & $2.35 \times 10^{-2}-4.49 \times 10^{-1}$ & $2.5 \eta_{T}$ & Implied from [58] \\
$f_{F}, \quad f_{M}$ & Normal & $8.5 \times 10^{-1}-9.5 \times 10^{-1}$ & 0.9 & Assumed \\
$g_{F}, \quad g_{M}$ & Gamma & $8.5 \times 10^{-1}-9.5 \times 10^{-1}$ & 0.9 & Assumed \\
$\delta_{F}, \quad \delta_{M}$ & Normal & $5.8 \times 10^{-2}-7.68 \times 10^{-2}$ & $6.849 \times 10^{-2}$ day $^{-1}$ & Implied from [20] \\
$\epsilon_{F}, \quad \epsilon_{M}$ & Uniform & $2.5 \times 10^{-1}-9.5 \times 10^{-1}$ & 0.8 & {$[59]$} \\
$c_{M}$ & Uniform & $4 \times 10^{-1}-8 \times 10^{-1}$ & 0.6 & Assumed \\
$c_{F}$ & Uniform & $1 \times 10^{-1}-3 \times 10^{-1}$ & 0.15 & Assumed \\
$\kappa_{F}, \quad \kappa_{M}$ & Gamma & $1.86 \times 10^{-1}-4.54 \times 10^{-1}$ & 0.3 & {$[58]$} \\
$\nu_{F}, \quad \nu_{M}$ & Gamma & $1-1.4$ & 1.2 & Assumed \\
$\xi_{F}, \quad \xi_{M}$ & Gamma & $3.15 \times 10^{-1}-7.39 \times 10^{-1}$ & 0.5 & Assumed \\
$\Lambda_{F}, \quad \Lambda_{M}$ & - & - & $1.0203 \times 10^{3}$ day $^{-1}$ & {$[11,57]$} \\
$\theta_{F}, \quad \theta_{M}$ & - & - & 0.9 & Assumed \\
$\gamma_{F}, \quad \gamma_{M}$ & Normal & $6 \times 10^{-4}-7.6436 \times 10^{-4}$ & $6.8 \times 10^{-4}$ day $^{-1}$ & Implied from [58] \\
\hline \hline
\end{tabular}

Table 3: Number of new cases of infections for various control strategies using different weights

\begin{tabular}{|l|l|l|l|}
\hline \hline$\omega_{9}, \omega_{10}, \omega_{11}, \omega_{12}$ & $c_{F}^{*}=c_{M}^{*}=0$ & $\tau_{F}^{*}=\tau_{M}^{*}=0$ & $\tau_{F}^{*}, \tau_{M}^{*}, c_{F}^{*}, c_{M}^{*}$ \\
\hline \hline $10^{1}$ & $6.3217 \times 10^{5}$ & $1.2927 \times 10^{6}$ & $3.5408 \times 10^{5}$ \\
\hline $10^{2}$ & $6.3217 \times 10^{5}$ & $1.2952 \times 10^{6}$ & $3.6376 \times 10^{5}$ \\
\hline $10^{3}$ & $6.3220 \times 10^{5}$ & $1.3188 \times 10^{6}$ & $4.1798 \times 10^{5}$ \\
\hline $10^{4}$ & $6.3447 \times 10^{5}$ & $1.5012 \times 10^{6}$ & $5.0647 \times 10^{5}$ \\
\hline $10^{5}$ & $6.8244 \times 10^{5}$ & $1.8544 \times 10^{6}$ & $5.4578 \times 10^{5}$ \\
\hline $10^{6}$ & $1.2124 \times 10^{6}$ & $1.8611 \times 10^{6}$ & $9.6992 \times 10^{5}$ \\
\hline $10^{7}$ & $2.0147 \times 10^{6}$ & $1.8611 \times 10^{6}$ & $1.6071 \times 10^{6}$ \\
\hline
\end{tabular}

